\title{
Scaling Relations for Intercalation Induced Damage in Electrodes
}

\author{
Chien-Fan Chen, ${ }^{1}$ Pallab Barai, ${ }^{1}$ Kandler Smith, ${ }^{2}$ Partha P. Mukherjee, ${ }^{1, z}$ \\ ${ }^{1}$ Department of Mechanical Engineering, Texas A\&M University, College Station, TX, USA \\ ${ }^{2}$ National Renewable Energy Laboratory, Golden, CO, USA
}

Revised manuscript submitted to

Electrochimica Acta

March, 2015

${ }^{\mathrm{z}}$ Correspondence: pmukherjee@tamu.edu (P. P. Mukherjee) 


\begin{abstract}
Mechanical degradation, owing to intercalation induced stress and microcrack formation, is a key contributor to the electrode performance decay in lithium-ion batteries (LIBs). The stress generation and formation of microcracks are caused by the solid state diffusion of lithium in the active particles. In this work, scaling relations are constructed for diffusion induced damage in intercalation electrodes based on an extensive set of numerical experiments with particle-level description of microcrack formation under disparate operating and cycling conditions, such as temperature, particle size, C-rate, and drive cycle. The microcrack formation and evolution in active particles is simulated based on a stochastic methodology. A reduced order scaling law is constructed based on an extensive set of data from the numerical experiments. The scaling relations include combinatorial constructs of concentration gradient, cumulative strain energy, and microcrack formation. The reduced order relations are further employed to study the influence of mechanical degradation on cell performance and validated against the high order model for the case of damage evolution during variable current vehicle drive cycle profiles.
\end{abstract}

Keywords: Lithium-ion battery electrode; mechanical degradation; scaling relation 


\section{Introduction}

Lithium-ion batteries (LIBs) are considered as the candidate of choice for vehicle electrification because of their favorable energy density and power capability [1-4]. Therefore, there is a significant interest to improve the performance, safety, and life of LIBs in recent years. Chemical and mechanical degradation in LIBs, such as microcrack formation in the electrode active particles due to diffusion induced stress $[5,6]$ and the solid-electrolyte interphase (SEI) formation [7, 8], cause a significant bearing on the LIB life and performance deterioration in terms of capacity fade and impedance rise $[9,10]$.

In recent years, stress generation and microcrack formation in LIB electrode active particles have received significant attention [11-13]. The evolution of microcracks with cycling is one cause of mechanical degradation in LIBs. In reality, the microcrack in the active particle either can form during the fabrication process or by diffusion induced stress (DIS) during operation $[14,15]$. In LIBs, the DIS is related to the concentration gradient of Li within the particle during the delithiation/lithiation process. The microcracks form when tensile stress inside the active material exceeds the fracture threshold. The initial flaws induced during fabrication can start to propagate or new microcracks can form inside the active material because of DIS [16]. The microcrack formation hinders the lithium diffusion inside the active particle and causes the capacity fade and impedance rise in LIB electrodes [10, 17]. Microcracks due to diffusion induced stress also impact chemical degradation due to the solid electrolyte interphase (SEI) formation and cell cycle life [18]. In previous studies, the orientation of initial pre-existing cracks [19] and particle size [20-22] was shown to influence the probability and magnitude of microcrack formation during the lithiation and delithiation process. The critical size of the initial imperfection and its influence on propagation has also been investigated [23, 24]. 
Scaling analysis and reduced order models are often used to reduce the complexity of governing equations in simulation and generalize material properties, which can reduce the computational cost. The scaling analysis has been used to reduce the complexity of the governing equations of vanadium redox flow batteries [25] and generalize the expression of the transport properties of $\mathrm{LiPF}_{6}$-based electrolytes [26]. The reduced order models of the lithiumion battery also have been introduced to alleviate the computational cost [27-29]. For the damage evolution in materials, scaling analysis has been used to interpret and better understand damage evolution in brittle [30] and ductile [31] materials. To simulate microcrack formation using lattice-based models, the continuum media were described as a set of elastic bonds with randomly distributed failure threshold [32]. Even though fracture surfaces for different brittle materials are never smooth, a universality in the roughness exponent exists [33, 34]. Qualitative estimation of the universal fracture surface roughness exponent can be conducted using latticebased models [35].

To the best of our knowledge, no scaling methodology for damage evolution in LIB electrodes has been established. Because the formation of microcracks inside the active particle is affected by the strain energy, which is induced by the lithium diffusion and is proportional to the concentration gradient inside the active particle, the correlation between concentration gradient, cumulative strain energy (CSE), and microcrack formation is worth studying. Moreover, although the microcrack formation has been successfully predicted from our previous work [36], the computational expense is still an obstacle we need to overcome. Reduced order models obtained from data-driven scaling analysis of diffusion induced damage can significantly decrease the computational cost. 
In this study, systematic simulations have been implemented by using the diffusion induced damage model developed by Barai and Mukherjee [36] to probe the concentration distribution, CSE, and microcrack formation during the single discharge process and drive cycles. Because the microcrack formation is strongly dependent on temperature [37], delithiation/lithiation rate, and particle size [10,38], these operating conditions are chosen for our numerical simulation experiments. According to the simulation results from the single discharge process, the relations between (i) CSE and concentration gradient and (ii) CSE and microcrack formation can be found as a function of particle size and operating condition (i.e., temperature and C-rate) by using the data-driven scaling method (see Figure 1 (a)). The relations can reduce the complexity of the diffusion induced damage model developed by Barai and Mukherjee [36]. The relations are further implemented in the electrochemical model of lithiumion batteries to study the influence of mechanical damage on the cell performance. In addition, the scaling laws, developed using constant current discharge numerical simulation experiments, are validated versus the higher order model for drive cycle charge/discharge profiles representing a more complex operating condition. From the investigation of damage evolution during the drive cycle, we observe that besides delithiation/lithiation rate, temperature, and particle size, the drive pattern also has an influence on the microcrack formation.

\section{Method and Theory}

\subsection{Diffusion Induced Damage}

The lithium diffusion inside the active particle induces the displacement of atoms and causes the accumulation of strain energy inside the material. When the strain energy stored in the material exceeds its fracture threshold, the microcracks form. The externally applied diffusion induced load is a function of the concentration gradient. Therefore, the CSE, which is the total 
energy released due to the evolution of the microcrack, is also proportional to the concentration gradient inside the active particle. Since the formation of microcracks hinders the diffusion of lithium inside the active particle, the effective solid phase diffusivity decreases with the increase of microcrack density $\left(f_{b b}\right)$. The decrease of effective solid phase diffusivity further increases the concentration gradient inside the active particle. The increase of concentration gradient leads to additional strain energy that forms more microcracks. The cascade process causes the microcrack propagation and capacity fade of the cell. According to the mechanism of microcrack formation, it is worth finding the relation between (i) concentration gradient and CSE and (ii) CSE and microcrack density. Moreover, because the microcrack formation is affected by the C-rate (reaction current density), temperature $T$, and particle size $D$ [10], the relations between concentration gradient, CSE, and microcrack should also be a function of C-rate, temperature, and particle size.

\section{$\underline{\text { 2.2 Diffusion Induced Damage Model }}$}

In this study, we used a stochastic methodology of simulate the diffusion induced damage developed by Barai and Mukherjee [36] to probe the concentration gradient, cumulative strain energy, and microcrack density during the delithiation process of active particles. The detail of this model is briefly described below. The model is based on a random lattice spring formalism coupled with solid state diffusion in active particles. By solving the generalized diffusion equation, derived from the Helmholtz free energy equation [39, 40], the distribution of lithium concentration can be simulated.

$$
\frac{\partial c(\vec{r}, t)}{\partial t}=\vec{\nabla} \cdot\left[\underset{\tilde{\sim}}{D} \cdot \vec{\nabla} c(\vec{r}, t)+\frac{\delta V}{k_{B} T} c(\vec{r}, t) \underset{\sim}{D} \cdot \vec{\nabla} c(\vec{r}, t)-\frac{\delta V}{k_{B} T} c(\vec{r}, t) \underset{\sim}{D} \cdot \vec{\nabla} \sigma_{k k}\right]
$$


In Eq. (1), $c(\vec{r}, t)$ is the space- and time-dependent concentration of lithium, $\vec{r}$ is the spatial coordinate, $\mathrm{t}$ is the time, $\underset{\sim}{D}$ signifies the second order diffusion coefficient tensor, $\sigma_{k k}$ is the hydrostatic stress, $\delta$ is a material dependent constant, $k_{\mathrm{B}}$ is the Boltzmann's constant, $T$ signifies temperature, and $V$ is the atomic volume. Eq. (1) is a very general expression and it has been simplified based on certain assumptions. One should note that the effect of hydrostatic stress on the diffusion process is rather negligible and can be removed from the general form of the governing equation [41]. Since the anisotropy in the diffusivity term has not been taken into consideration, the $\underset{\sim}{D}$ tensor becomes a diagonal matrix, where the scalar value of the diagonal entries equals the diffusion coefficient $D_{s}$. For diffusion in isotropic graphite active materials, the diffusivity tensor can be simplified as a scalar with magnitude $D_{s}$. Incorporating the assumptions mentioned above, the updated form of Eq. (1) that has been solved to obtain the change in concentration profile is given below:

$$
\frac{\partial c(x, y, t)}{\partial t}=\frac{\partial}{\partial x}\left(D_{s}(x, y, t) \frac{\partial c(x, y, t)}{\partial x}\right)+\frac{\partial}{\partial y}\left(D_{s}(x, y, t) \frac{\partial c(x, y, t)}{\partial y}\right)
$$

Eq. (1a) was solved using the finite volume method. The flux due to the electrochemical reaction on the boundary of active particles can be expressed as

$$
\frac{\partial c(\vec{r}, t)}{\partial n}=\frac{i}{S F}
$$

where $i$ is the applied current and $S$ is the surface area of active particles in the electrode. The diffusion induced stress and evolution of fracture were estimated in a two-dimensional representative circular cross-section of the spherical active particle. In our study, a technique 
based on random spring model (RSM) has been used to estimate the initiation and accumulation of damage and fracture in the electrode [42, 43]. In the present study, the authors focus on lithium insertion and subsequent microcrack formation within the graphite electrode that is usually used as the anode active material. Since the failure of graphite occurs in a brittle fashion, the active material studied here is assumed to display brittle fracture behavior [44, 45]. The lattice network has a coordination number of six with triangular meshes in this study. The fracture threshold energy of each spring $\left(E_{t}\right)$ is distributed randomly with a normal distribution, where the mean values have been evaluated based on an energy equivalency scheme [43, 46, 47]. By adopting the Li concentration profile inside the active particle, the stress distribution inside the active particle can be evaluated from the quasi-static equilibrium equation.

$$
\frac{\partial \sigma_{i j}}{\partial x_{j}}+F_{i}=\rho \ddot{\vec{u}}_{i}=0
$$

with $u_{i}=\bar{u}_{i}$ on $S_{u}$ and $\sigma_{i j} n_{j}=t_{i}=\bar{t}$ on $S_{t}$. Here, $F_{i}$ is the body force vector which is assumed to be zero for this particular problem $\left(F_{i}=0\right), \sigma_{i j}$ is the stress tensor, $u_{i}\left(x_{i}\right)$ is the space dependent displacement vector, $\bar{u}_{i}$ is the prescribed displacement on the boundary, $x_{i}$ signifies the spatial coordinates, $\sigma_{i j}$ is the stress tensor on the boundary, $n_{j}$ is the outer normal direction on the domain boundary, $S_{u}$ is that portion of the boundary on which the displacement prescribed boundary condition is specified, $S_{t}$ is that portion of the boundary on which traction prescribed boundary condition is specified, $t_{i}$ is the traction force, and $\bar{t}_{i}$ is the prescribed traction force on the boundary. Small strain small displacement analysis has been conducted here. 
The above-mentioned equations were discretized and solved by spring type elements, which display stiffness along both the axial and transverse directions. The local force versus displacement relation for the spring is [48],

$$
\left[\begin{array}{l}
f_{x 1} \\
f_{y 1} \\
f_{x 2} \\
f_{y 2}
\end{array}\right]=\left[\begin{array}{cccc}
k_{n} & 0 & -k_{n} & 0 \\
0 & k_{s} & 0 & -k_{s} \\
-k_{n} & 0 & k_{n} & 0 \\
0 & -k_{s} & 0 & k_{s}
\end{array}\right]\left[\begin{array}{l}
u_{x 1} \\
u_{y 1} \\
u_{x 2} \\
u_{y 2}
\end{array}\right]
$$

where $\vec{f}$ is the local force vector, $\vec{u}$ is the local displacement vector, $k_{n}$ is the axial spring stiffness, and $k_{s}$ is the shear stiffness of each spring. Diffusion induced displacement $\Delta u^{d}$ and force $\vec{f}^{d}$ that acts on each of the lattice spring elements are defined as[19, 49],

$$
\begin{aligned}
& \Delta u^{d}=\Gamma \cdot \Delta c \cdot l \\
& \vec{f}^{d}=\left[k_{l}\right] \cdot \Delta \vec{u}^{d}
\end{aligned}
$$

Stress generated due to the diffusion of $\mathrm{Li}$ is incorporated as an axial displacement of the spring. In Eqs. (5) and (6), $\Gamma$ signifies the volume expansion coefficient (with the unit of $\mathrm{m}^{3} / \mathrm{mol}$ ) and $\Delta c$ represents the local concentration gradient $\left(\Delta c=c(x, t)-c_{a v g}(t)\right.$, where $c_{\text {avg }}(t)$ represents the volume averaged concentration of the entire spherical particle), $l$ is the length of the spring type element, and $\left[k_{l}\right]$ is the local stiffness matrix. Microcrack formation is determined in terms of the energy threshold criterion. The energy stored in each spring can be expressed as

$$
\psi=\frac{1}{2} \vec{f} \cdot \vec{u}
$$

where $\vec{f}$ and $\vec{u}$ are the local force and displacement vectors for each of the springs. The spring is irreversibly removed from the lattice network when the strain energy in a spring exceeds the 
fracture threshold $\left(\psi>\psi_{t}\right)$. As mentioned, the fracture threshold for each of the springs $\left(\psi_{t}\right)$ is assigned randomly with a normal distribution around the mean fracture threshold. After one bond is broken, the lattice network is re-equilibrated before breaking subsequent bonds. In this numerical experiment, the temperature affects the conservation of lithium (see Eq. (1)) and the diffusivity $D_{s .}$. The temperature dependent diffusivity follows the Arrhenius relation as

$$
D_{s}(T)=D_{0, s} \exp \left[\frac{E_{a}}{R}\left(\frac{1}{T_{r e f}}-\frac{1}{T}\right)\right]
$$

Figure 1 (b) shows an example of simulation results from the diffusion induced damage model. Concentration and microcrack distribution are shown in the representative active particle. The microcrack density can be approximately defined as the ratio between the broken bonds (black dots) and the total number of springs inside the particle. As shown in Figure 1 (b), during the delithiation process, the microcrack forms on the surface of the particle due to the large concentration gradient.

\subsection{Dimensionless Parameters}

In order to better correlate the relation between CSE, concentration gradient, and microcrack density, the dimensionless radius $\bar{R}$, temperature $\bar{T}$, cumulative strain energy $\overline{C S E}$, and concentration gradient $\bar{C}$ are introduced as follows

$$
\begin{aligned}
& \bar{R}=\frac{R}{R_{\text {ref }}}, R_{r e f}=12.5 \mu \mathrm{m} \\
& \bar{T}=\left(\frac{T}{T_{g}}-1\right), T_{g}=238 \mathrm{~K}
\end{aligned}
$$




$$
\begin{gathered}
\overline{C S E}=\frac{C S E}{E_{\text {threshold }}}, E_{\text {threshold }}=2 \mathrm{~J} / \mathrm{m}^{2} \\
\bar{C}=\frac{C_{\text {avg }}-C_{\text {surf }}}{C_{\text {max }}}, C_{\max }=31833 \mathrm{~mol} / \mathrm{m}^{3}
\end{gathered}
$$

where $T_{g}$ is the glass transition temperature of the binder, $E_{\text {threshold }}$ is the mean fracture threshold energy, $C_{a v g}$ is the average bulk concentration in the particle, $C_{\max }$ is the maximum bulk concentration one particle can store, and $C_{\text {surf }}$ is the average surface concentration in the particle. As mentioned, the relations between CSE, concentration gradient, and microcrack density strongly depend on temperature, C-rate, and particle size. Therefore, one possibility for the relations between CSE, concentration gradient, and microcrack can be assumed as Eqs. (13) and (14). The microcrack density in Eq. (13) is defined as the ratio between the broken springs and the total number of springs inside the lattice network.

$$
\bar{C} \text { and Microcrack Density }=a \cdot \overline{\operatorname{CSE}}^{b} \cdot M^{-1}
$$

where $M$ is the scaling factor, which is based on the temperature, particle radius, and C-rate.

$$
M=\bar{T}^{c} \cdot \bar{R}^{d} \cdot(\text { C-rate })^{e}
$$

The schematic diagram of finding the relation between CSE, concentration gradient, and microcrack density (Eq. (13)) is as shown in Figure 1 (a). With the diffusion induced damage model described in the previous subsection, one set of operating conditions (i.e., combination of C-rate, temperature, and particle size) can have one set of results, which includes CSE, concentration gradient, and microcrack density. By conducting a systematic study of variable possible operating conditions (see Table 3), we can get a map between operating conditions and results. By tuning the scaling factor $M$ (Eq. (14)), the massive sets of results can converge to four 
relations, which can be expressed by Eqs. (13) and (14). The four relations are the relation between CSE and concentration gradient when (i) $T>0^{\circ} \mathrm{C}\left(f_{1}\right)$ and (ii) $T \leq 0^{\circ} \mathrm{C}\left(f_{2}\right)$ and the relation between CSE and microcrack density when (iii) $T>0^{\circ} \mathrm{C}\left(f_{3}\right)$ and (iv) $T \leq 0^{\circ} \mathrm{C}\left(f_{4}\right)$.

\subsection{Electrochemical-Mechanical Coupled Model}

The relation between microcrack density $\left(f_{b b}\right)$, concentration gradient $(\bar{C})$, and $\overline{C S E}$ shown in Eqs. (13) and (14) can be coupled with the electrochemical model to study the influence of diffusion induced damage on cell performance. Using reduced order relations can decrease the computational expense thereby decreasing simulation time without compromising on accuracy. The schematic diagram of the electrochemical-mechanical coupled model is presented in Figure 1 (c). The details of the electrochemical model used to simulate cell performance are described in Appendix A. From the electrochemical model, using the calculated surface solid-phase concentration $c_{s}$ (from Eqs. (A5) and (A6)), the $\overline{C S E}$ can be obtained from Eq. (13) and the scaling factor $M$ (see Table 4). In the scaling factor $M$, the C-rate is taken from the electrochemical model reaction current density. The $1 \mathrm{C}$ delithiation rate corresponds to the reaction current density of $i=2.11 \mathrm{~A} / \mathrm{m}^{2}$. The $\overline{C S E}$ can be further used to calculate the corresponding microcrack density $f_{b b}$ from Eq. (13) and scaling factor $M$ (see Table 4). According to our previous work, the relation between microcrack density and effective solidphase diffusivity $D_{s}^{\text {eff }}$ can be expressed as [50]

$$
D_{s}^{\text {eff }}=D_{0, s} \cdot\left(1-f_{b b}\right)^{\gamma}
$$

Here, $D_{0}$ signifies the diffusivity of the pristine anode active particle, $f_{b b}$ is the microcrack density and $\gamma$ is the power-law exponent (value adopted from Barai et al.[50]). The effective 
solid-phase diffusivity obtained from the microcrack density affects the cell performance since the solid phase concentration $c_{s}$ is highly dependent on the effective solid-phase diffusivity.

\section{Results and Discussion}

\section{$\underline{\text { 3.1 Scaling Analysis of Delithiation Process }}$}

In this section, we present the correlation between CSE, concentration gradient, and microcrack density during a single delithiation process under different operating conditions with varied particle size. A series of simulations, which used the high order diffusion induced damage model, were implemented to tune the parameters in Eqs. (13) and (14). The parameters used in the diffusion induced damage model are listed in Table 1 . The simulated operating conditions and particle sizes are listed in Table 3. The 1C delithiation rate corresponds to

operation at $1.656 \mathrm{~A}\left(i=2.11 \mathrm{~A} / \mathrm{m}^{2}\right)$. The concentration distribution, CSE, and number of microcracks were recorded whenever the microcrack formed. In the single delithiation process, the applied current is maintained constant. Under the same applied current, the delithiation process was stopped when the surface concentration reached zero. In this study, we considered an electrode-only model, specifically for a representative anode active particle. Therefore, all the parameters in the following diffusion induced damage model refer to a typical graphite active material.

\subsubsection{Cumulative Strain Energy and Concentration Gradient}

As mentioned in the previous section, due to the imbalance between the delithiation rate and solid phase diffusion rate, there is a concentration gradient inside the active particle that generates strain energy inside the active material. The CSE is calculated by summing the strain energy released due to the microcrack formation inside the active particle. The relation between CSE and concentration gradient can be affected by the temperature, C-rate, and particle size. 
Figure 2 (a) and Figure 3 (a) show the influence of particle size on the relation between CSE and concentration gradient when $T=20^{\circ} \mathrm{C}$ and $-10^{\circ} \mathrm{C}$. The $5.5 \mu \mathrm{m}$ particle has a lower maximum CSE because it has less microcrack formation compared to larger particles. From the results, we can observe that, with the same CSE, the concentration gradient increases with the particle size. The reason is because the length for lithium to diffuse from the center to the surface is longer in larger particles. The larger concentration gradient leads to faster lithium depletion at the particle surface [51]. Figure 2 (c) and Figure 3 (c) show the influence of delithiation rate (i.e., C-rate) on the relation between CSE and concentration gradient when $T=$ $20^{\circ} \mathrm{C}$ and $-10^{\circ} \mathrm{C}$. Similarly, we can observe that the concentration gradient increases with the Crate, which is due to the high lithium depletion rate on the surface of particles under high C-rate. Figure 4 presents the effect of temperature. From the results, under the same CSE, the concentration gradient increases with a decrease in operating temperature. The influence of temperature is mainly on the solid phase diffusivity. According to Eq.(8), the solid phase diffusivity decreases with temperature. The decrease of diffusivity further hinders the diffusion of lithium from center to surface, and hence increases the concentration gradient.

With this systematic study, we found that if we multiply the concentration gradient with a scaling factor $M$, the data converge as shown in Figures 2, 3, and 4 . The scaling factor $M$ for the relation of CSE and concentration gradient under different temperatures, C-rates, and particle sizes are listed in Table 4. Figure 2 (b) and (d) suggests that the data from different particle sizes and C-rates converge after multiplying a scaling factor $M_{1}$ to the concentration gradient when $T=20^{\circ} \mathrm{C}$. Similarly, under subzero temperature, the data converge after multiplying a scaling factor $M_{2}$ to the concentration gradient as shown in Figure 3 (b) and (d). The effect of temperature also can be generalized by the scaling factor as suggested in Figure 4 (b) and (d). It 
is worth noting that the scaling factor is different when $T>0^{\circ} \mathrm{C}$ and $T<0^{\circ} \mathrm{C}$ as shown in Table 4. From the scaling factor, we can infer the contribution of C-rate, temperature, and particle size to the relation between CSE and concentration gradient from the order of C-rate, temperature, and particle size. The higher the order, the larger the influence. Because the variation of solid phase diffusivity $\left(d D_{s} / d T\right)$ is higher when $T>0^{\circ} \mathrm{C}\left(M_{1}\right)$ compared to $T \leq 0^{\circ} \mathrm{C}\left(M_{2}\right)$, according to Eq. (8), the temperature has a larger influence on concentration gradient when $T>0^{\circ} \mathrm{C}\left(M_{1}\right)$ compared to $T \leq 0^{\circ} \mathrm{C}\left(M_{2}\right)$. Similarly, due to the low solid phase diffusivity, the variation of the C-rate has a larger influence on the concentration gradient when $T \leq 0^{\circ} \mathrm{C}\left(M_{2}\right)$ compared to $T>$ $0^{\circ} \mathrm{C}\left(M_{1}\right)$

\subsubsection{Cumulative Strain Energy and Microcrack Density}

From the mechanism of microcrack formation, when the strain energy exceeds the threshold energy, the microcrack will form. Therefore, the microcrack inside the active particle should be proportional to the CSE. In this subsection, we present the relation between CSE and microcrack density with the same approach of finding the relation between CSE and concentration gradient. Figure 5 (a) and Figure 6 (a) show the effect of particle size on the relation between CSE and microcrack density under different temperature. In Figure 5 (a), we can see that the particle size of $12.5 \mu \mathrm{m}$ has the smallest maximum microcrack density compared to the other two particle sizes. This is because the long diffusion distance for lithium and the resultant high concentration gradient decrease the discharge time (i.e., the discharge stops when the surface concentration reaches zero). Moreover, the microcrack density decreases with the increase of particle size when the CSE is the same. This result can be explained from the perspective of the concentration gradient and the distribution of strain energy. As mentioned in the previous section, the bonds break when the strain energy exceeds the threshold energy. In 
other words, if the strain energy is below the threshold energy, the bond will sustain. CSE is defined as the summation of energy released due to the rupture of the connecting bonds. The cumulative strain energy can be written as,

$$
C S E=\frac{1}{2} \sum_{e=1}^{n_{b b}} \vec{f}_{e} \cdot \vec{u}_{e} \approx \frac{1}{2} k_{a} \cdot \Omega \cdot(\Delta c) \cdot l \cdot u_{a} \cdot n_{b b}
$$

where $n_{b b}$ is the total number of broken bonds, and $\vec{f}_{e}$ and $\vec{u}_{e}$ signify the force and displacement in each of the broken bonds, respectively. Also, $k_{a}, \Omega, l$ and $u_{a}$ denote axial stiffness, partial molar volume, length of each element, and axial displacement, respectively. These four parameters and variables can be approximated to remain almost constant. The last variable $\Delta c$ signifies the concentration gradient, which differs based on particle size, operating condition, and ambient temperature. Let's denote the cumulative strain energy for a large particle as $C S E_{l}$ and for a small particle as $C S E_{s}$.

$$
\operatorname{CSE}_{l}=\frac{1}{2} k_{a} \cdot \Omega \cdot(\Delta c)_{l} \cdot l \cdot u_{a} \cdot n_{l} \text { and } \operatorname{CSE}_{s}=\frac{1}{2} k_{a} \cdot \Omega \cdot(\Delta c)_{s} \cdot l \cdot u_{a} \cdot n_{s}
$$

Here, $(\Delta c)_{l}$ and $(\Delta c)_{s}$ correspond to the concentration gradient for large and small particles, respectively. Similarly, $n_{l}$ and $n_{s}$ signify the number of broken bonds for the larger and the smaller particles, respectively. For this particular case, where cumulative strain energy released from the larger and smaller particle is same, we can write $C S E_{l}=C S E_{s}$, comparing it with the previous equation, $(\Delta c)_{l} \cdot n_{l}=(\Delta c)_{s} \cdot n_{s}$. Concentration gradient in a larger particle is greater in magnitude than the concentration gradient for a small particle, which implies that $(\Delta c)_{l}>(\Delta c)_{s}$. As a result, $n_{l}<n_{s}$, which implies that for a constant value of CSE, microcrack density in the 
larger particle is less than the microcrack density within the smaller particle. Figure 5 (c) and Figure 6 (c) show the influence of C-rate on the relation between CSE and microcrack density when $T=20^{\circ} \mathrm{C}$ and $-10^{\circ} \mathrm{C}$. Similar to Figure 5 (a) and Figure 6 (a), under the same CSE, the microcrack density decreases with the increase of the C-rate, which is similar to the case for different particle size. During discharge under a high C-rate, due to the larger concentration gradient, smaller microcrack density will be observed under the constraint of constant CSE. Therefore, the particle discharge under high C-rate has less microcrack density compared to the one discharge under low C-rate. Figure 7 (a) and (c) shows the influence of temperature on the relation between CSE and microcrack density. The results suggest that the microcrack density decreases with decreasing temperature under the same CSE, which can also be explained by the variation in the concentration gradient. The concentration gradient in the particle operating at low temperature is much higher than the one operating at high temperature. As a result, under constant cumulative strain energy, particles operating at low temperature will have smaller microcrack density as compared to active particles at higher ambient temperature conditions.

Similar to the relation between CSE and concentration gradient, the data converges when multiplying the microcrack density with a scaling factor $M$, as shown in Figures 5, 6, and 7. The scaling factors for the relations between CSE and microcrack density are listed in Table 4. In Figures 5 and 6, the effects of C-rate and particle size can be generalized by using scaling factor $M_{3}$ and $M_{4}$ when $T=20^{\circ} \mathrm{C}$ and $0^{\circ} \mathrm{C}$ respectively. For the influence of temperature, the data also converges after multiplying the scaling factor by the microcrack density as shown in Figure 7. This scaling result is similar to the relationship between CSE and the concentration gradient. The scaling factor varies with the temperature and can be used to infer which factor has a higher influence on damage. Since the variation of solid phase diffusivity $\left(d D_{s} / d T\right)$ is higher when $T$ 
$>0^{\circ} \mathrm{C}\left(M_{3}\right)$ compared to $T \leq 0^{\circ} \mathrm{C}\left(M_{4}\right)$ according to Eq. (8), the temperature has a larger influence on concentration gradient when $T>0^{\circ} \mathrm{C}\left(M_{3}\right)$ compared to $T \leq 0^{\circ} \mathrm{C}\left(M_{4}\right)$. When $T \leq$ $0^{\circ} \mathrm{C}$, the effect of temperature decreases, but different from the relation between CSE and concentration, the influence of C-rate was not enhanced by the low temperature. Moreover, by comparing the order in the scaling factor for the relation between CSE/concentration gradient (0.14) and CSE/microcrack density (0.28), we can observe that the temperature, C-rate, and particle size have a higher impact on the relation of CSE/microcrack density than the relation of CSE/concentration.

With the introduction of the scaling factor, we can find the parameters in Eq. (13) by fitting the data obtained from the simulations as shown in Figure 8. The data in Figure 8 is from the simulation conditions listed in Table 3. From the curve fitting, the suggested parameters for Eq. (13) are listed in Table 4.

\subsection{Influence of Mechanical Damage on Cell Performance}

Once the scaling relations for the evolution of fracture have been established, they can be implemented into the pseudo 2D lithium ion battery model which has been developed based on porous electrode theory [50]. The parameters used in the pseudo 2D lithium ion battery model are listed in Table 2 and the detail of model is presented in Appendix A. Evolution of mechanical degradation along the thickness of the anode during a single discharge process has been investigated here. Because of the mechanical degradation, the mass transport resistance within the solid phase increases. To capture this phenomenon, reduction in effective diffusivity of the solid phase has been modeled according to a power law relation as shown in Eq. (15). Performance curves obtained at different C-rates (1C and 3C) for an anode of radius $8.5 \mu \mathrm{m}$ has been shown in Figure 9 (a). The total amount of capacity fade due to microcrack formation is 
also reflected in the performance curve. The overall mechanical degradation at $1 \mathrm{C}$ and $3 \mathrm{C}$ is demonstrated in Figure 9 (b). At lower rates of operation, less concentration gradient develops within the active particles, which give rise to less damage evolution. Reduced mechanical degradation at smaller rates of operation results in a negligible capacity fade. Enhanced microcrack density is observed close to the separator as compared to the current collector because the higher magnitude of reaction current density is experienced by the particles near the separator.

It has already been established that smaller sized particles experience less microcrack formation. Figure 9(c) estimates the impacts of different particle size on the mechanical degradation induced capacity fade at a high rate of operation. Very small particles of size 5.5 $\mu \mathrm{m}$ experience almost negligible capacity fade due to mechanical degradation. However, as reported in Figure 9 (d), anode particles of radius $5.5 \mu \mathrm{m}$ can experience around 1.5\% to 2\% mechanical degradation (specifically close to the separator). Slightly larger sized particles of radius $8.5 \mu \mathrm{m}$ experience close to $3 \%$ microcrack density, which has the potential to give rise to approximately 3.5\% capacity fade. Large particles of size $12.5 \mu \mathrm{m}$ are mostly affected by the mechanical degradation. They not only experience severe microcrack formation as high as $4 \%$, but also significant capacity-fade, which can exceed $10 \%$ in a single discharge process. In the computational program, if the surface SOC in the cathode or anode goes above 1.0 or below 0.0 , the code is forcefully stopped. In Figure 9 (c) the performance curve for $12.5 \mu \mathrm{m}$ active particle with mechanical degradation stops at around $3.1 \mathrm{~V}$ because the surface SOC within anode goes out of bounds. Along the thickness of the anode, smaller particles experience enhanced damage evolution close at the separator (see Figure 9 (d)). However, large particles of radius $12.5 \mu \mathrm{m}$ experience almost uniform damage evolution throughout anode. This uniform damage evolution 
can be attributed to the movement of the maximum reaction current front from the separator to the current collector over the course of the discharge process [50].

\subsection{Scaling Analysis of Drive Cycle}

In addition to the scaling study for the single delithiation process, the microcrack formation during the drive cycle is also discussed in this paper. One should note that because the C-rate is not constant during the drive cycle, the pattern for the relation between CSE and the concentration gradient is irregular. Therefore, the relation between CSE and the concentration gradient is not discussed in this subsection. This subsection considers drive cycles for three different electric-drive vehicle applications: plug-in hybrid electric vehicle (PHEV), hybrid electric vehicle (HEV), and battery electric vehicle (BEV). The three were simulated using the high order diffusion induced damage model [36]. The scaling equations obtained from the previous section (see Eq. (13) and Table 4) have been used to reproduce the relation between CSE and microcrack density for drive cycle.

Figure 10 shows the pattern of C-rate during different drive cycles. The average discharge

rate, average charge rate, discharge fraction, and charge fraction are shown in Table 5. The HEV drive cycle has a higher discharge/charge rate compared to PHEV and BEV drive cycles. However, because the fraction of the discharge/charge period of the HEV drive cycle is nearly 50\%/50\%, the average C-rate is lower than the PHEV and BEV drive cycles.

Figures 11, 12, and 13 present the influence of temperature and particle size on the microcrack formation for PHEV, BEV, and HEV drive cycles, respectively. The time evolution of the concentration gradient varies frequently due to the variable C-rate of the different drive cycles. For the PHEV drive cycle shown in Figure 11 (a) and (d), because the variation of C-rate is large, we can see a large fluctuation of the concentration gradient. In contrast, the smaller C- 
rate variation during the $\mathrm{BEV}$ drive cycle causes less fluctuation of concentration gradient compared to the PHEV drive cycle as shown in Figure 12 (a) and (d). For the HEV drive cycles, we can see large fluctuations of the concentration gradient as shown in Figure 13 (a) and (d). The difference in the concentration gradient of the different drive cycles greatly affects the average microcrack density. The small concentration gradients of the BEV drive cycle (Figure 12 (a) and (d)) results in microcrack density about 10 times smaller than the other two drive cycles.

For the PHEV drive cycle, Figure 11 (a) shows that the concentration gradient increases with the decrease of temperature. This is due to the temperature-dependent characteristic of diffusivity as shown in Eq. (8). Since the concentration gradient is high under low operating temperature, the particle has a higher microcrack density than operating under higher temperature as shown in Figure 11 (b). The relation between CSE and microcrack density shown in Figure 11 (c) is similar to the single delithiation process, in which low temperature has a lower microcrack density under the same value of CSE. This is caused by the difference in concentration gradient as explained in the previous section. Figure 11 (d) shows the time evolution of the concentration gradient in different particle sizes during the PHEV drive cycle. The results suggest that the concentration gradient increases with the particle size. The variation of concentration gradient is due to the change in diffusion length when the particle size is different. The change of concentration gradient affects the microcrack density as shown in Figure 11 (e). Figure 11 (f) presents the relation between CSE and microcrack density for different particle sizes, with similar trends as the single delithiation process. The microcrack density decreases with the increased particle size under the same CSE. By comparing the time evolution of the concentration gradient in Figure 11 (a) and (d), and the time evolution of 
microcrack density in Figure 11 (b) and (e), we discover the difference in microcrack density is mainly due to the peak concentration gradient, observable as step changes in microcrack density in Figure 11 (e), at the approximate times of $t=50,100$, and 300 seconds. One should note that since the concentration gradient is large in the $12.5 \mu \mathrm{m}$ particle, the sudden increase of microcrack density shifts from $t=300$ seconds to 200 seconds. At these times, due to the large peak in the concentration gradient, the microcrack density has a significant increase. Moreover, it is worth noting that, although there is a large fluctuation after 500 seconds, the microcrack density does not increase. This is because the concentration gradient during 300 to 500 seconds saturates the microcrack density, and the lower concentration gradient after 500 seconds has no potential to form new microcracks. This phenomenon is even obvious when the particle size is $12.5 \mu \mathrm{m}$ and $T=20^{\circ} \mathrm{C}$, that the microcrack density saturated after 200 seconds.

Figure 12 shows the effects of temperature and particle size on the microcrack formation for the BEV drive cycle. Similar to the PHEV drive cycle, the concentration gradient increases with the decrease of temperature and the increase of particle size, as shown in Figure 12 (a) and (d). The relation between CSE and microcrack density shown in Figure 12 (c) and (f) also has the same trend as the singe delithiation process and PHEV drive cycle. Similarly, we can also observe a sudden increase of microcrack density in the $6 \mu \mathrm{m}$ and $8.5 \mu \mathrm{m}$ particle when $\mathrm{t}=300$ seconds due to the large impulse of the concentration gradient. The phenomenon is less obvious because of the low concentration gradient. The microcrack density only has a significant increase when $\mathrm{T}=-10^{\circ} \mathrm{C}$.

Figure 13 shows the effects of temperature and particle size on the microcrack formation for the charge sustaining HEV drive cycle. The trend of time evolution in the concentration gradient, time evolution of microcrack density, and the CSE/microcrack density relations show 
the same trend as for the PHEV and BEV drive cycles. However, the influence of temperature and particle size on the concentration gradient is insignificant as shown in Figure 13 (a) and (d), which is because of the discharge/charge period fraction during the drive cycles. The nearly $50 \% / 50 \%$ period fraction alleviates the influence of diffusion length and solid phase diffusivity on the concentration gradient. Due to the insignificant variation of the concentration gradient, the microcrack formation during the HEV drive cycle is mainly caused by the sudden pulse of concentration gradient at the beginning of the drive cycle and $t=100$ seconds. It is worth noting that although the HEV drive cycle was operating under the highest discharge/charge rate, due to the nearly 50\%/50\% charge/discharge period fraction, the HEV drive cycle has less mechanical damage compared to the PHEV drive cycle.

Figure 14 shows the reproduction of the relation between CSE and microcrack density by using the scaling laws found in the previous section. The relation is as shown in Eq. (13) and Table 4. During the drive cycle operation, whenever the microcrack formed, the corresponding cumulative strain energy and C-rate were recorded. For reproduction, the recorded C-rate was used to calculate the scaling factor $M$ (see Table 4). With the scaling factor and CSE, we can evaluate the microcrack density from Eq. (13). When calculating the scaling factor, because not every C-rate in the drive cycle has contributed to the microcrack formation according to the results shown in Figures 11, 12 and 13, we averaged the C-rate every 5 time steps of microcrack formation, and used the average C-rate to calculate the scaling factor. In general, the scaling law (reduced order model) in Eq. (13) reasonably captures the microcrack growth trend. The fitting equation shows good agreement with the results of the BEV drive cycle. However, we still can see fluctuations in the reproduction of the PHEV and HEV drive cycle data, because (i) it is hard 
to pick the exact average C-rate, which has contributed to microcrack formation, and (ii) the formation location of microcracks is different during delithiation and lithiation processes [10].

\section{Conclusion}

The influence of temperature, particle size, and delithiation rate on the damage evolution in an active particle of LIB electrodes has been investigated using a high order diffusion induced damage model. The evolution of microcrack formation was quantified both for a single delithiation process and for several drive cycles with highly variable C-rate profiles.

From the single delithiation simulations, the temperature, particle size, and C-rate show significant influence on the relation between (i) CSE and concentration gradient and (ii) CSE and microcrack density. For the relation between CSE and concentration gradient, the results suggest that, under the same value of CSE, the concentration gradient increases with (i) decrease of temperature, (ii) increase of particle size, and (iii) increase of C-rate. In contrast, for the relation between CSE and microcrack density, the microcrack density increases with (i) increase of temperature, (ii) decrease of particle size, and (iii) decrease of C-rate under the same value of CSE. According to the results, we introduced a scaling factor $M$, which can collapse the data from different operating conditions. Scaling law expressions for the relationships between CSE, concentration gradient, and microcrack density were found by fitting data from the high order diffusion induced damage model. From the data-drive scaling process, different temperature conditions $\left(\mathrm{T}>0^{\circ} \mathrm{C}\right.$ or $T \leq 0^{\circ} \mathrm{C}$ ) suggested different scaling factors $M$, which means that the contribution of operation factors (i.e., temperature, particle size, and C-rate) varied with the temperature. The reduced-order equations were coupled with the electrochemical model to study the influence of mechanical damage on cell performance. According to the results, the capacity fade is larger when the microcrack density is higher. Further, the capacity fade is directly 
proportional to the size of the electrode active particles and discharging rates. Another important observation is that the microcrack density is higher in the region near the separator as compared to the region near the current collector since the current density is higher near the separator.

In addition to the observation of the single delithiation process, we also investigated the damage evolution during the drive cycle. Three kinds of drive cycles (HEV, PHEV, and BEV) were tested in this study. The results quantified the microcrack formation dependence on temperature, particle size, and C-rate. Since PHEV and HEV applications operate under a higher C-rate than the BEV application, the model predicts those applications will experience higher microcrack density. During the drive cycle, the microcrack was saturated after operation over time. After saturation, new microcracks can only form when the average concentration gradient exceeds its previous peak value. In addition, the scaling law expression for the relation between CSE and microcrack density found from the single delithiation process has successfully predicted the damage evolution during different drive cycles. This result validates our proposed approach for reduced order modeling of mechanical damage evolution in LIB active material particles.

\section{Acknowledgements}

Financial support from National Renewable Energy Laboratory (NREL) as a research contract to Texas A\&M University is gratefully acknowledged. KS acknowledges funding provided by the U.S. Department of Energy, Vehicles Technologies Office, Energy Storage Program (program manager, Brian Cunningham). Assistance from Eric Wood at NREL for providing vehicle drive cycle profiles and from Bhaskar Vajipeyajula at Texas A\&M University at the initial stage of this work are also gratefully acknowledged. 


\section{Appendix A}

The reduced-order equations present in Table 4 can be coupled with the electrochemical model to study the influence of diffusion induced damage on the cell performance. The cell performance presented in this study is obtained by solving the following conservation equations.

The charge conservation in solid phase and electrolyte is expressed as Eq. (A1) and Eq. (A2) respectively.

$$
\begin{gathered}
\nabla\left(\sigma_{s}^{e f f} \nabla \phi_{s}\right)-j=0 \\
\nabla\left(\kappa^{e f f} \nabla \phi_{e}+\kappa_{D}^{e f f} \nabla \ln c_{e}\right)+j=0
\end{gathered}
$$

The effective ionic conductivity $\kappa^{\text {eff }}$ and diffusional ionic conductivity $\kappa_{D}^{\text {eff }}$ are calculated using the diffusivity of the electrolyte phase $D_{e}$ and the electrolyte concentration $c_{e}$.

$$
\kappa^{e f f}=\frac{z^{2} F^{2} D_{e}^{e f f} C_{e, i n i}}{R T} \text { and } \kappa_{D}^{e f f}=z F D_{e}^{e f f}
$$

The mass conservation in the electrolyte and solid active particles is expressed as Eq. (A4) and Eq. (A5) respectively.

$$
\begin{aligned}
\varepsilon \frac{\partial c_{e}}{\partial t} & =\nabla\left(D_{e}^{e f f} \nabla c_{e}\right)+\frac{1-t_{+}}{F} j \\
\frac{\partial c_{s}}{\partial t} & =\frac{1}{r^{2}} \frac{\partial}{\partial r}\left(D_{s}^{e f f} r^{2} \frac{\partial c_{s}}{\partial r}\right)
\end{aligned}
$$

The boundary condition of solving mass conservation in active particles is 


$$
-\left.D_{s} \frac{\partial c_{s}}{\partial r}\right|_{r=R_{i}}=\frac{i}{F} \text { and }\left.\frac{\partial c_{s}}{\partial r}\right|_{r=0}=0
$$

The charge transfer kinetics can be calculated by Butler-Volmer equation.

$$
i=i_{0}\left[\exp \left(\frac{\alpha_{a} F}{R T} \eta\right)-\exp \left(-\frac{\alpha_{c} F}{R T} \eta\right)\right]
$$

where the overpotential $\eta$ and exchange current density $i_{0}$ are

$$
\begin{gathered}
\eta=\phi_{s}-\phi_{e}-U\left(c_{s}\right) \\
i_{0}=k c_{s}^{\alpha_{c}} C_{e}^{\alpha_{a}}\left(c_{s, \max }-c_{s}\right)^{\alpha_{a}}
\end{gathered}
$$

The relation between the reaction current density on particle surface $i$ and volumetric current density $j$ is given by

$$
j=a_{s} i
$$

The open-circuit potential $U$ for anode and cathode are determined from empirically derived functions of Li-ion surface concentration, which can be expressed as below.

$$
\begin{aligned}
& U_{n}=0.13966+0.6892 e^{-49.20361 x_{n}}+0.41903 e^{-254.40067 x_{n}} \\
& -e^{49.97886 x_{n}-43.37888}-0.028221 \cdot \arctan \left(22.523 x_{n}-3.65328\right) \\
& -0.01308 \cdot \arctan \left(28.34801 x_{n}-13.4396\right) \\
& U_{p}=4.04596+e^{-42.30027 x_{p}+16.56714} \\
& -0.0488 \cdot \arctan \left(50.01833 x_{p}-26.48897\right) \\
& -0.05447 \cdot \arctan \left(18.99678 x_{p}-12.32362\right)-e^{78.24095 x_{p}-78.68074}
\end{aligned}
$$

where $x_{p}$ and $x_{n}$ are the surface state of charge (SOC) which can be defined as 


$$
S O C=x_{i}=\frac{C_{\text {surf }, i}}{C_{\text {max }, i}}, i=n, p
$$

The effective electrolyte diffusivity can be calculated from Bruggeman relation as eq. (A11).

$$
D_{e}^{e f f}=D \cdot \varepsilon^{1.5}
$$

\section{Appendix B}

In the porous electrode theory reported in this article, it has been assumed that the current that flows between the solid and the electrolyte phase are estimated based on Butler-Volmer reaction kinetics (Eq. (A7)). The Butler-Volmer equation is derived from the Nernst equation, which estimates the potential of a material as a function of concentration[52]. The Nernst equation has been developed for perfect materials free from any mechanical stresses. However, in an actual porous electrode, the solid active particles experience severe stress, which arises from not only the transport of species, but also from the interaction between adjacent particles[53]. To incorporate the effect of mechanical stress, the Nernst equation has been modified[54]:

$$
E=E^{0}-\frac{R T}{\zeta n F} \ln \left(c_{L i}\right)
$$

Here, $E$ is the electrochemical potential, $E_{0}$ signifies a reference potential, $R$ is the universal gas constant, $T$ indicates absolute temperature in Kelvin scale, $n$ is the number of electrons associated with each reaction, $F$ is the Faraday's constant, $c_{L i}$ indicates the concentration of lithium and, finally, $\zeta$ signifies the correction parameter to the Nernst equation. Incorporation of the term $\zeta$ is an empirical modification to the anodic component only. Following this direction, 
a similar empirical modification to the anodic current of the Butler-Volmer equation can be suggested:

$$
i=i_{0}\left[\exp \left(\frac{\zeta \alpha_{a} F}{R T} \eta\right)-\exp \left(-\frac{\alpha_{c} F}{R T} \eta\right)\right]
$$

The impact of different values of the correction parameter $\zeta$ on the cell performance (voltage vs. capacity curve) as well as mechanical degradation, will be analyzed in the present section.

Figure B1 shows the effect of different values of $\zeta$ ranging from $1 / 3$ to 3.0. The cell has been operated at $1 \mathrm{C}$ and average particle sizes of $5 \mu \mathrm{m}$ and $8.5 \mu \mathrm{m}$ have been assumed in the cathode and anode, respectively. Increasing the value of $\zeta$ from 1.0 to 2.0 and then to 3.0 results in a reduction of the activation polarization because of a smaller magnitude of charge transfer resistance. The voltage increases slightly, but there is no significant improvement in the cell capacity, because the capacity is governed by mass transport limitations. On the other hand, as the correction parameters $\zeta$ decreases from 1.0 to $1 / 2$ and finally to $1 / 3$, the charge transfer resistance increases. As a result, the cell voltage drops and reaches the cutoff voltage earlier than usual, leading to an overall reduction in cell capacity. No mechanical degradation has been assumed in these simulations.

It has already been reported earlier that mechanical degradation is less during operation at lower rates, and increases significantly for high rate application (see Fig. 9(b)). Incorporation of the correction parameter $\zeta$ changes the charge transfer resistance, and subsequently the reaction current. Since the reaction current determines the rate of operation for individual particles, $\zeta$ will definitely have some impact on the overall mechanical degradation experienced by the electrodes. Figure B2 analyzes the effect of correction parameter on the overall mechanical degradation and subsequent the impact on cell performance during operation at 3C. Figure B2(a) 
indicates that increasing the value of $\zeta$ (from 1.0 to 2.0) reduces the charge transfer resistance and results in the smaller magnitude of activation polarization. Similarly, reducing the value of $\zeta$ (from 1.0 to $1 / 2$ ) results in higher kinetic polarization and smaller magnitude of cell voltage, which leads to reduction in capacity. These features are similar to that observed in Figure B1 during operation at 1C. Figure B2(b) depicts the amount of mechanical degradation along the thickness of anode for values of $\zeta$ ranging between 2.0 and $1 / 2$. . Higher values of the correction parameter result in smaller charge transfer resistance and larger magnitude of reaction rate. Hence, most microcracks are observed for $\zeta=2.0$. As the value of $\zeta$ decreases, smaller magnitude of reaction current density results in less evolution of microcracks. From figure B2(b) it is clear that maximum mechanical degradation is observed close to the separator. There also exists a location somewhere along the thickness of anode where minimum microcrack formation is observed.

\section{References}

[1] R. Wagner, N. Preschitschek, S. Passerini, J. Leker, and M. Winter, "Current research trends and prospects among the various materials and designs used in lithium-based batteries," Journal of Applied Electrochemistry, vol. 43, pp. 481-496, 2013/05/01 2013.

[2] M. S. Whittingham, "Lithium Batteries and Cathode Materials," Chemical Reviews, vol. 104, pp. 4271-4302, 2004/10/01 2004.

[3] B. L. Ellis, K. T. Lee, and L. F. Nazar, "Positive Electrode Materials for Li-Ion and LiBatteries†," Chemistry of Materials, vol. 22, pp. 691-714, 2010/02/09 2010.

[4] J. M. Tarascon and M. Armand, "Issues and challenges facing rechargeable lithium batteries," Nature, vol. 414, pp. 359-367, Nov 152001.

[5] F. Hao and D. Fang, "Diffusion-Induced Stresses of Spherical Core-Shell Electrodes in Lithium-Ion Batteries: The Effects of the Shell and Surface/Interface Stress," Journal of The Electrochemical Society, vol. 160, pp. A595-A600, January 1, 20132013.

[6] S. Kalnaus, K. Rhodes, and C. Daniel, "A study of lithium ion intercalation induced fracture of silicon particles used as anode material in Li-ion battery," Journal of Power Sources, vol. 196, pp. 8116-8124, 10/1/ 2011.

[7] D. Aurbach, "The Role of Surface Films on Electrodes in Li-ion Batteries," in Advances in Lithium-Ion Batteries, W. A. v. Schalkwijk and B. Scrosati, Eds., ed New York: Academic/Plenem Publishers, 2002, p. 7. 
[8] J. W. Jiang and J. R. Dahn, "Effects of solvents and salts on the thermal stability of LiC6," Electrochimica Acta, vol. 49, pp. 4599-4604, Oct 152004.

[9] C.-F. Chen and P. P. Mukherjee, "Probing the morphological influence on solid electrolyte interphase and impedance response in intercalation electrodes," Physical Chemistry Chemical Physics, vol. 17, pp. 9812-9827, 2015.

[10] C.-F. Chen, P. Barai, and P. P. Mukherjee, "Diffusion Induced Damage and Impedance Response in Lithium-Ion Battery Electrodes," Journal of The Electrochemical Society, vol. 161, pp. A2138-A2152, January 1, 20142014.

[11] Y.-T. Cheng and M. W. Verbrugee, "Diffusion-Induced Stress, Interfacial Charge Transfer, and Criteria for Avoiding Crack Initiation of Electrode Particles.," Journal of The Electrochemical Society, vol. 157, pp. A508 - A516, 2010.

[12] S. Golmon, K. Maute, and M. L. Dunn, "Numerical modeling of electrochemicalmechanical interactions in lithium polymer batteries," Computers and Structures, vol. 87, pp. 1567 - 1579, 2009.

[13] K. Takahashi and V. Srinivasan, "Examination of Graphite Particle Cracking as a Failure Mode in Lithium-Ion Batteries: A Model-Experimental Study," Journal of The Electrochemical Society, vol. 162, pp. A635-A645, January 1, 20152015.

[14] S. J. Harris, R. D. Deshpande, Y. Qi, I. Dutta, and Y.-T. Cheng, "Mesopores inside electrode particles can change the Li-ion transport mechanism and diffusion-induced stress," Journal of Materials Research, vol. 25, pp. 1433 - 1440, 2010.

[15] S. De, B. Suthar, D. Rife, G. Sikha, and V. R. Subramanian, "Efficient Reformulation of Solid Phase Diffusion in Electrochemical-Mechanical Coupled Models for Lithium-Ion Batteries: Effect of Intercalation Induced Stresses," Journal of the Electrochemical Society, vol. 160, pp. A1675-A1683, 2013.

[16] J. Lemaitre and J. L. Chaboche, Mechanics of solid materials: Cambridge University Press, 1990.

[17] P. Arora, R. E. White, and M. Doyle, "Capacity fade mechanisms and side reactions in Lithium - Ion batteries," Journal of The Electrochemical Society, vol. 145, pp. 3647 3667, 1998.

[18] R. Deshpande, M. Verbrugge, Y. T. Cheng, J. Wang, and P. Liu, "Battery Cycle Life Prediction with Coupled Chemical Degradation and Fatigue Mechanics," Journal of the Electrochemical Society, vol. 159, pp. A1730-A1738, 2012.

[19] R. Grantab and V. B. Shenoy, "Location - and Orientation - Dependent Crack Propagation in Cylindrical Graphite Electrode Particles," Journal of The Electrochemical Society, vol. 158, pp. A948 - A954, 2011.

[20] B. J. Dimitrijevic, K. E. Aifantis, and K. Hackl, "The influence of particle size and spacing on the fragmentation of nanocomposite anodes for Li batteries," Journal of Power Sources, vol. 206, pp. 343 - 348, 2012.

[21] Y.-T. Cheng and M. W. Verbrugee, "The influence of surface mechanics on diffusion induced stresses within spherical nanoparticles," Journal of Applied Physics, vol. 104, p. 083521, 2008.

[22] C. K. Chan, H. Peng, G. Liu, K. McIlwrath, X. F. Zhang, R. A. Huggins, et al., "Highperformance lithium battery anodes using silicon nanowires," Nature Nanotechnology, vol. 3, pp. 31 - 35, 2008.

[23] K. Zhao, M. Pharr, J. J. Vlassak, and Z. Suo, "Fracture of electrodes in lithium-ion batteries caused by fast charging," Journal of Applied Physics, vol. 108, p. 073517, 2010. 
[24] W. H. Woodford, Y.-M. Chiang, and W. C. Carter, "'Electrochemical Shock" of Intercalation Electrodes: A Fracture Mechanics Analysis," Journal of The Electrochemical Society, vol. 157, pp. A1052 - A1059, 2010.

[25] M. Vynnycky, "Analysis of a model for the operation of a vanadium redox battery," Energy, vol. 36, pp. 2242-2256, 4// 2011.

[26] L. O. Valoen and J. N. Reimers, "Transport properties of LiPF6-based Li-ion battery electrolytes," Journal of the Electrochemical Society, vol. 152, pp. A882-A891, 2005.

[27] X. Li, M. Xiao, and S.-Y. Choe, "Reduced order model (ROM) of a pouch type lithium polymer battery based on electrochemical thermal principles for real time applications," Electrochimica Acta, vol. 97, pp. 66-78, 5/1/ 2013.

[28] V. Senthil Kumar, "Reduced order model for a lithium ion cell with uniform reaction rate approximation," Journal of Power Sources, vol. 222, pp. 426-441, 1/15/ 2013.

[29] T.-S. Dao, C. P. Vyasarayani, and J. McPhee, "Simplification and order reduction of lithium-ion battery model based on porous-electrode theory," Journal of Power Sources, vol. 198, pp. 329-337, 1/15/ 2012.

[30] J. A. Astrom, "Statistical models of brittle fragmentation," Advances in Physics, vol. 55, pp. 247-278, May-Jun 2006.

[31] M. Zaiser, "Scale invariance in plastic flow of crystalline solids," Advances in Physics, vol. 55, pp. 185-245, Jan-Apr 2006.

[32] L. Dearcangelis, S. Redner, and H. J. Herrmann, "A Random Fuse Model for Breaking Processes," Journal De Physique Lettres, vol. 46, pp. L585-L590, 1985.

[33] A. Hansen and J. Schmittbuhl, "Origin of the universal roughness exponent of brittle fracture surfaces: Stress-weighted percolation in the damage zone," Physical Review Letters, vol. 90, Jan 312003.

[34] J. Krim and J. O. Indekeu, "Roughness Exponents - a Paradox Resolved," Physical Review E, vol. 48, pp. 1576-1578, Aug 1993.

[35] P. K. V. V. Nukala, S. Zapperi, M. J. Alava, and S. Simunovic, "Anomalous roughness of fracture surfaces in 2D fuse models," International Journal of Fracture, vol. 154, pp. 119-130, Nov 2008.

[36] P. Barai and P. P. Mukherjee, "Stochastic Analysis of Diffusion Induced Damage in Lithium-Ion Battery Electrodes," Journal of the Electrochemical Society, vol. 160, pp. A955-A967, 2013.

[37] K. An, P. Barai, K. Smith, and P. P. Mukherjee, "Probing the Thermal Implications in Mechanical Degradation of Lithium-Ion Battery Electrodes," Journal of The Electrochemical Society, vol. 161, pp. A1058-A1070, January 1, 20142014.

[38] P. Barai and P. P. Mukherjee, "Mechano-Electrochemical Model for Acoustic Emission Characterization in Intercalation Electrodes," Journal of the Electrochemical Society, vol. 161, pp. F3123-F3136, 2014.

[39] M. V. Paukshto, "Diffusion-induced stresses in solids," International Journal of Fracture, vol. 97, pp. 227-236, 1999/04/01 1999.

[40] I. V. Belova and G. E. Murch, "Thermal and diffusion nduced stresses in crystalline solids," Journal of Applied Physics, vol. 77, pp. 127-134, 1995.

[41] X. Zhang, W. Shyy, and A. M. Sastry, "Numerical simulation of intercalation-induced stress in Li-ion battery electrode particles (vol 154, pg A910, 2007)," Journal of the Electrochemical Society, vol. 154, pp. S21-S21, 2007. 
[42] P. K. V. V. Nukala, S. Zapperi, and S. Simunovic, "Statistical properties of fracture in a random spring model," Physical Review E, vol. 71, p. 066106, 2005.

[43] P. Barai, R. Sampath, P. K. V. V. Nukala, and S. Simunovic, "Scaling of surface roughness in perfectly plastic disordered media," Physical Review E, vol. 82, Nov 19 2010.

[44] R. W. Davidge and G. Tappin, "The Effective Surface Energy of Brittle Materials," Journal of Materials Science, vol. 3, pp. 165-173, Mar 1968.

[45] Y. Qi, L. G. Hector, C. James, and K. J. Kim, "Lithium Concentration Dependent Elastic Properties of Battery Electrode Materials from First Principles Calculations," Journal of the Electrochemical Society, vol. 161, pp. F3010-F3018, 2014.

[46] P. K. V. V. Nukala, S. Zapperi, M. J. Alava, and S. Šimunović, "Crack roughness in the two-dimensional random threshold beam model," Physical Review E, vol. 78, p. 046105, 10/13/ 2008.

[47] S. Zapperi, P. K. Nukala, and S. Simunovic, "Crack roughness and avalanche precursors in the random fuse model," Phys Rev E Stat Nonlin Soft Matter Phys, vol. 71, p. 026106, Feb 2005.

[48] G.-F. Zhao, J. Fang, and J. Zhao, "A 3D distinct lattice spring model for elasticity and dynamic failure," International Journal for Numerical and Analytical Methods in Geomechanics, vol. 35, pp. 859 - 885, 2011.

[49] R. D. Cook, D. S. Malkus, M. E. Plesha, and R. J. Witt, Concepts and Applications of Finite Element Analysis, Fourth Edition ed.: John Wiley and Sons (Asia) Pte Ltd, 2002.

[50] P. Barai, K. Smith, C. F. Chen, G. H. Kim, and P. P. Mukherjee, "Reduced Order Modeling of Mechanical Degradation Induced Performance Decay in Lithium-Ion Battery Porous Electrodes," Journal of the Electrochemical Society, vol. 162, pp. A1751A1771, 2015.

[51] K. C. Smith, P. P. Mukherjee, and T. S. Fisher, "Columnar order in jammed LiFePO4 cathodes: ion transport catastrophe and its mitigation," Physical Chemistry Chemical Physics, vol. 14, pp. 7040-7050, 2012.

[52] H. H. Girault, Analytical and Physical Electrochemistry. New York, USA: EPFL Press, 2004.

[53] M. D. Levi, D. Aurbach, and J. Maier, "Electrochemically driven first-order phase transitions caused by elastic responses of ion-insertion electrodes under external kinetic control," Journal of Electroanalytical Chemistry, vol. 624, pp. 251-261, Dec 12008.

[54] A. V. Churikov, A. V. Ivanishchev, A. V. Ushakov, and V. O. Romanova, "Diffusion aspects of lithium intercalation as applied to the development of electrode materials for lithium-ion batteries," Journal of Solid State Electrochemistry, vol. 18, pp. 1425-1441, May 2014.

[55] M. Guo, G. H. Kim, and R. E. White, "A three-dimensional multi-physics model for a Liion battery," Journal of Power Sources, vol. 240, pp. 80-94, Oct 152013.

[56] W. B. Gu and C. Y. Wang, "Thermal-electrochemical coupled modeling of a lithium-ion cell," Proceedings of the Electrochemical Society, vol. 99, p. 748, 2000. 


\section{List of Tables}

Table 1. List of parameters used to solve the diffusion induced damage model (see Ref. [36]).

Table 2. List of parameters used to solve the pseudo 2D lithium ion battery model. All the parameters shown below have been adopted from Guo et al. (see Ref. [55]) and Gu and Wang (see Ref. [56])

Table 3. Combinatorial matrix of operating and drive cycle conditions

Table 4. Scaling constructs in Eq. (13)

Table 5. Statistical data of drive cycle

\section{List of Figures}

Figure 1. (a) The schematic diagram of data-driven approach toward reduced order relations. With the scaling factor $M$, the massive number of results can be reduced to four relations. (b) Example of microcrack formation inside a representative active particle, which includes the microcrack and concentration distribution. (c) Schematic diagram for the electrochemicalmechanical coupled model.

Figure 2. Relation between cumulative strain energy and concentration gradient for (a) different particle sizes at $1 \mathrm{C}$-rate and $20^{\circ} \mathrm{C}$ before multiplying by the scaling factor; (b) different particle sizes at $1 \mathrm{C}$-rate and $20^{\circ} \mathrm{C}$ after multiplying by the scaling factor; (c) different C-rates at $20^{\circ} \mathrm{C}$ before multiplying by the scaling factor with the particle diameter of $8.5 \mu \mathrm{m}$; and (d) different $\mathrm{C}$ rates at $20^{\circ} \mathrm{C}$ after multiplying by the scaling factor with the particle diameter of $8.5 \mu \mathrm{m}$.

Figure 3. Relation between cumulative strain energy and concentration gradient for (a) different particle sizes at $1 \mathrm{C}$-rate and $-10^{\circ} \mathrm{C}$ before multiplying by the scaling factor; (b) different particle sizes at $1 \mathrm{C}$-rate and $-10^{\circ} \mathrm{C}$ after multiplying by the scaling factor; (c) different C-rates at $-10^{\circ} \mathrm{C}$ before multiplying by the scaling factor with the particle diameter of $8.5 \mu \mathrm{m}$; and (d) different Crates at $-10^{\circ} \mathrm{C}$ after multiplying by the scaling factor with the particle diameter of $8.5 \mu \mathrm{m}$.

Figure 4. Relation between cumulative strain energy and concentration gradient for different operation temperatures at $1 \mathrm{C}$-rate with the particle diameter of $8.5 \mu \mathrm{m}$. (a) $\mathrm{T} \leq 0^{\circ} \mathrm{C}$, before multiplying by the scaling factor. (b) $\mathrm{T} \leq 0^{\circ} \mathrm{C}$, after multiplying by the scaling factor. (c) $\mathrm{T}>$ $0^{\circ} \mathrm{C}$, before multiplying by the scaling factor. (d) $\mathrm{T}>0^{\circ} \mathrm{C}$, after multiplying by the scaling factor.

Figure 5. Relation between cumulative strain energy and microcrack density for (a) different particle sizes at $2 \mathrm{C}$-rate and $20^{\circ} \mathrm{C}$ before multiplying by the scaling factor; (b) different particle sizes at $2 \mathrm{C}$-rate and $20^{\circ} \mathrm{C}$ after multiplying by the scaling factor; (c) different C-rates at $20^{\circ} \mathrm{C}$ before multiplying by the scaling factor with the particle diameter of $8.5 \mu \mathrm{m}$; and (d) different Crates at $20^{\circ} \mathrm{C}$ after multiplying by the scaling factor with the particle diameter of $8.5 \mu \mathrm{m}$. 
Figure 6. Relation between cumulative strain energy and microcrack density for (a) different particle sizes at $1 \mathrm{C}$-rate and $-10^{\circ} \mathrm{C}$ before multiplying by the scaling factor; (b) different particle sizes at $1 \mathrm{C}$-rate and $-10^{\circ} \mathrm{C}$ after multiplying by the scaling factor; (c) different C-rates at $-10^{\circ} \mathrm{C}$ before multiplying by the scaling factor with the particle diameter of $8.5 \mu \mathrm{m}$; and (d) different Crates at $-10^{\circ} \mathrm{C}$ after multiplying by the scaling factor with the particle diameter of $8.5 \mu \mathrm{m}$.

Figure 7. Relation between cumulative strain energy and concentration gradient for different operation temperatures at 2 C-rate with the particle diameter of $12.5 \mu \mathrm{m}$. (a) $\mathrm{T} \leq 0^{\circ} \mathrm{C}$, before multiplying by the scaling factor. (b) $\mathrm{T} \leq 0^{\circ} \mathrm{C}$, after multiplying by the scaling factor. (c) $\mathrm{T}>$ $0^{\circ} \mathrm{C}$, before multiplying by the scaling factor. (d) $\mathrm{T}>0^{\circ} \mathrm{C}$, after multiplying by the scaling factor.

Figure 8. The scaling result for (a) relation between cumulative strain energy and concentration gradient when $\mathrm{T}>0^{\circ} \mathrm{C}$; (b) relation between cumulative strain energy and concentration gradient when $\mathrm{T} \leq 0^{\circ} \mathrm{C}$; (c) relation between cumulative strain energy and microcrack density when $\mathrm{T}>$ $0^{\circ} \mathrm{C}$; and $(\mathrm{d})$ relation between cumulative strain energy and microcrack density when $\mathrm{T} \leq 0^{\circ} \mathrm{C}$. The dashed line shows the fitting result.

Figure 9. (a) Performance at different C-rates with and without taking damage evolution into account. Capacity fade is due to mechanical degradation is more prominent at higher C-rates. (b) Distribution of microcrack density at the end of the discharge process of different C-rates. Higher mechanical degradation is observed at larger C-rates. (c) Performance curves for three different particle sizes with and without taking the effect of microcrack evolution into account. Enhanced capacity fade is observed in large sized particles. (d) Evolution of mechanical degradation is more for large sized particles. For small particles $(5.5 \mu \mathrm{m}$ and $8.5 \mu \mathrm{m})$, larger microcrack density evolves near the separator. However, large sized particles experience almost uniform mechanical degradation throughout the anode thickness.

Figure 10. (a) Variation of C-rate with time for the PHEV drive cycle. (b) Variation of C-rate with time for the BEV drive cycle. (c) Variation of C-rate with time for the HEV drive cycle.

Figure 11. $(a-c)$ The influence of temperature on the PHEV drive cycle with particle diameter of $8.5 \mu \mathrm{m}$. (a) The time evolution of the concentration gradient. (b) The time evolution of microcrack density. (c) The relation between microcrack density and CSE. (d-f) The influence of particle size on the PHEV drive cycle at $T=20^{\circ} \mathrm{C}$. (d) The time evolution of the concentration gradient. (e) The time evolution of microcrack density. (f) The relation between microcrack density and CSE.

Figure 12. $(\mathrm{a}-\mathrm{c})$ The influence of temperature on the BEV drive cycle with particle diameter of $8.5 \mu \mathrm{m}$. (a) The time evolution of the concentration gradient. (b) The time evolution of microcrack density. (c) The relation between microcrack density and CSE. (d-f) The influence of particle size on the BEV drive cycle at $T=20^{\circ} \mathrm{C}$. (d) The time evolution of the concentration gradient. (e) The time evolution of microcrack density. (f) The relation between microcrack density and CSE.

Figure 13. $(\mathrm{a}-\mathrm{c})$ The influence of temperature on the HEV drive cycle with particle diameter of $8.5 \mu \mathrm{m}$. (a) The time evolution of the concentration gradient. (b) The time evolution of 
microcrack density. (c) The relation between microcrack density and CSE. (d-f) The influence of particle size on the HEV drive cycle at $T=20^{\circ} \mathrm{C}$. (d) The time evolution of the concentration gradient. (The results of particle size $6 \mu \mathrm{m}$ and $8.5 \mu \mathrm{m}$ almost overlap). (e) The time evolution of microcrack density. (f) The relation between microcrack density and CSE.

Figure 14. Comparison of the relation between microcrack density and CSE between high order diffusion induced damage model and reduced order relation in Eq. (13). (a) PHEV drive cycle with $8.5 \mu \mathrm{m}$ particle under $-10^{\circ} \mathrm{C}$. (b) BEV drive cycle with $8.5 \mu \mathrm{m}$ particle under $-10^{\circ} \mathrm{C}$. (c) HEV drive cycle with $8.5 \mu \mathrm{m}$ particle under $-10^{\circ} \mathrm{C}$.

Figure: B1. Effect of the correction parameter $\zeta$ on the voltage vs. capacity performance curves during operation at $1 \mathrm{C}$. As the correction parameter increases from 1.0 to 3.0, the kinetic resistances decreases and the voltage curve moves upward, but to a very small magnitude. However, as $\zeta$ decreases to $1 / 2$ or $1 / 3$, the polarization due to charge transfer increases significantly, and the voltage drops by a significant amount.

Figure: B2. Effect of the correction parameter $\zeta$ on the mechanical degradation during high rate operation. This simulation has been conducted at $3 \mathrm{C}$ for graphite anode and NMC cathode type active materials. (a) Voltage vs. capacity curves at 3C for three different values of $\zeta(1.0,2.0$ and $1 / 2$ ). Mechanical degradation is significant at higher rates of operation. (b) Microcrack density along the thickness of anode for three different values of $\zeta$ at the end of discharge at 3C. 


\title{
List of Symbols
}

\author{
$a_{s} \quad$ specific surface area \\ $c_{e} \quad$ space and time dependent lithium ion concentration within electrolyte \\ $c_{s} \quad$ space and time dependent lithium concentration within the solid phase \\ $D_{e}^{\text {eff }} \quad$ effective diffusivity of the electrolyte phase \\ $D_{e} \quad$ diffusivity of the electrolyte phase \\ $D_{s}^{\text {eff }} \quad$ effective diffusivity of the solid phase \\ $D_{s} \quad$ diffusivity of the solid pahse \\ $F \quad$ faraday's constant \\ I applied current \\ $i_{0} \quad$ exchange current density \\ $i \quad$ reaction current density \\ $k_{n} \quad$ spring stiffness along the axial direction \\ $k_{s} \quad$ spring stiffness along the shear direction \\ $L_{a} \quad$ thickness of anode \\ $L_{c} \quad$ thickness of cathode \\ $R \quad$ universal gas constant \\ $R_{S} \quad$ outer radius of the solid active particle \\ $t_{+} \quad$ transference number \\ $T$ Temperature \\ $U \quad$ open circuit potential \\ $z \quad$ charge number of the diffusing species
}

\section{Greek}

$\begin{array}{cl}\alpha_{a} & \text { anodic transfer coefficient } \\ \alpha_{c} & \text { cathodic transfer coefficient } \\ \gamma & \text { an exponent to capture the effect of microcrack on diffusivity } \\ \varepsilon & \text { porosity of the electrolyte phase } \\ \eta & \text { overpotential for positivie or negative electrode } \\ \kappa & \text { conductivity of electrolyte } \\ \kappa^{\text {eff }} & \text { effective conductivity of electrolyte } \\ \kappa_{d} & \text { diffusional ionic conductivity } \\ \kappa_{d}^{\text {eff }} & \text { effective diffusional ionic conductivity } \\ \sigma & \text { conductivity of solid phase } \\ \sigma^{\text {eff }} & \text { effective conductivity of solid phase } \\ \phi_{e} & \text { electrolyte phase potential } \\ \phi_{s} & \text { solid phase potential }\end{array}$


Table 1. List of parameters used to solve the diffusion induced damage model (see Ref. [36]).

\begin{tabular}{lcc}
\multicolumn{1}{c}{ Name } & Units & Value \\
\hline Expansion coefficient $(\Gamma)$ & $\mathrm{m}^{3} / \mathrm{mol}$ & $1.14 \times 10^{-6}$ \\
Diffusion coefficient $(\underset{\sim}{D})$ & $\mathrm{m}^{2} / \mathrm{s}$ & $3.9 \times 10^{-14}$ \\
Anode particle surface area $(\mathrm{S})$ & $\mathrm{m}^{2}$ & 0.7824 \\
Axial direction spring stiffness & $\mathrm{kN} / \mathrm{m}$ & 88.1717 \\
Shear direction spring stiffness & $\mathrm{kN} / \mathrm{m}$ & 11.6688
\end{tabular}


Table 2. List of parameters used to solve the pseudo 2D lithium ion battery model. All the parameters shown below have been adopted from Guo et al. (see Ref. [55]) and Gu and Wang (see Ref. [56])

\begin{tabular}{lcccc}
\multicolumn{1}{c}{ Name } & Units & Anode & Separator & Cathode \\
\hline Length $\left(L_{a}, L_{s}, L_{c}\right)$ & $\mu \mathrm{m}$ & 130 & 26 & 130 \\
Porosity $(\varepsilon)$ & - & 0.357 & 0.41 & 0.444 \\
Solid phase conductivity $(\sigma)$ & $\mathrm{S} / \mathrm{m}$ & 100 & - & 3.8 \\
Maximum surface concentration $\left(c_{\max }\right)$ & $\mathrm{mol} / \mathrm{m}^{3}$ & 31833 & - & 51410 \\
Electrolyte diffusivity $\left(D_{e}\right)$ & $\mathrm{m}^{2} / \mathrm{s}$ & $7.5 \times 10^{-11}$ & $7.5 \times 10^{-11}$ & $7.5 \times 10^{-11}$ \\
Solid phase diffusivity $\left(D_{0, s}\right)$ & $\mathrm{m}^{2} / \mathrm{s}$ & $3.9 \times 10^{-14}$ & - & $1.0 \times 10^{-13}$ \\
Initial electrolyte concentration & $\mathrm{mol} / \mathrm{m}^{3}$ & 2000 & 2000 & 2000 \\
Transference number $\left(t_{+}\right)$ & - & 0.363 & - & 0.363
\end{tabular}


Table 3. Combinatorial matrix of operating and drive cycle conditions.

\begin{tabular}{c|lc}
\hline & Factor & Conditions \\
\hline \multirow{5}{*}{ Single Delithiation } & Temperature & {$[-10,-5,0,10,20,30]{ }^{\circ} \mathrm{C}$} \\
& C-rate & {$[1,2,3,4,5] \mathrm{C}$} \\
& Particle Diameter & {$[5.5,6,8.5,12.5] \mu \mathrm{m}$} \\
& (Total: 120 sets of operating conditions) \\
\hline \multirow{5}{*}{ Drive Cycles } & Temperature & {$[-10,0,20]^{\circ} \mathrm{C}$} \\
& Particle Diameter & {$[6,8.5,12.5] \mu \mathrm{m}$} \\
& Drive Pattern & {$[\mathrm{HEV}$, PHEV, BEV] }
\end{tabular}


Table 4. Scaling constructs in Eq. (13).

\begin{tabular}{cccc}
\hline Relation & a & b & M \\
\hline$\overline{C S E}$ and $\bar{C}\left(\mathrm{~T}>0^{\circ} \mathrm{C}\right)$ & 0.0061 & 0.465 & $M_{1}=\left[\frac{\text { C-Rate } \times \bar{R}}{\bar{T}^{2}}\right]^{-0.28}$ \\
$\overline{C S E}$ and $\bar{C}\left(\mathrm{~T} \leq 0^{\circ} \mathrm{C}\right)$ & 0.0072 & 0.275 & $M_{2}=\left[\frac{C-\text { Rate }{ }^{2} \times \bar{R}}{\bar{T}}\right]^{-0.28}$ \\
$\overline{C S E}$ and Microcrack Density $\left(\mathrm{T}>0^{\circ} \mathrm{C}\right)$ & 0.0015 & 0.657 & $M_{3}=\left[\frac{C-\text { Rate } \times \bar{R}}{\bar{T}^{2}}\right]^{0.14}$ \\
$\overline{C S E}$ and Microcrack Density $\left(\mathrm{T} \leq 0^{\circ} \mathrm{C}\right)$ & 0.0016 & 0.8443 & $M_{4}=\left[\frac{C-\text { Rate } \times \bar{R}}{\bar{T}}\right]^{0.14}$
\end{tabular}


Table 5. Statistical data of drive cycle.

\begin{tabular}{ccccc}
\hline Drive Cycle & $\begin{array}{c}\text { Average } \\
\text { Discharge Rate }\end{array}$ & $\begin{array}{c}\text { Average } \\
\text { Charge Rate }\end{array}$ & Discharge Fraction & Charge Fraction \\
\hline HEV & $2.66 \mathrm{C}$ & $2.04 \mathrm{C}$ & $44.5 \%$ & $55.5 \%$ \\
$\mathrm{PHEV}$ & $1.46 \mathrm{C}$ & $1.03 \mathrm{C}$ & $75.8 \%$ & $24.2 \%$ \\
$\mathrm{BEV}$ & $0.496 \mathrm{C}$ & $0.305 \mathrm{C}$ & $88 \%$ & $12 \%$
\end{tabular}




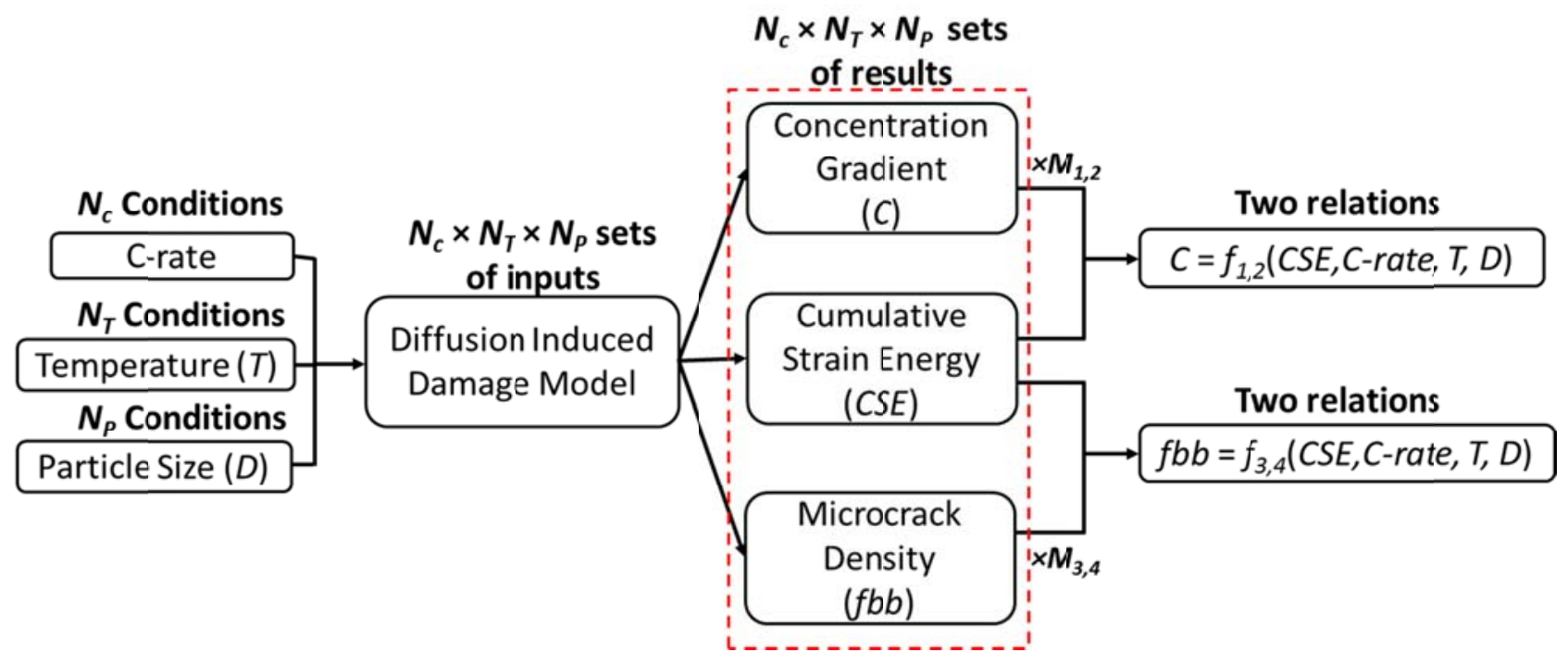

(a)

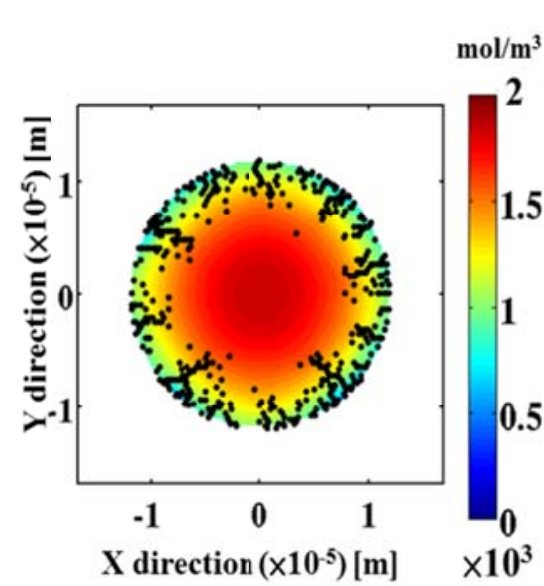

(b)
Mechanical Coupling

Microcrack Density

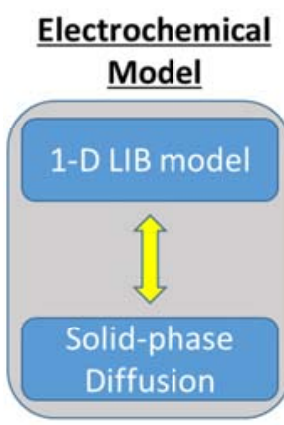

. Gradient

(c)

Figure 1. (a) The schematic diagram of data-driven approach toward reduced order relations. With the scaling factor $M$, the massive number of results can be reduced to four relations. (b) Example of microcrack formation inside a representative active particle, which includes the microcrack and concentration distribution. (c) Schematic diagram for the electrochemicalmechanical coupled model. 

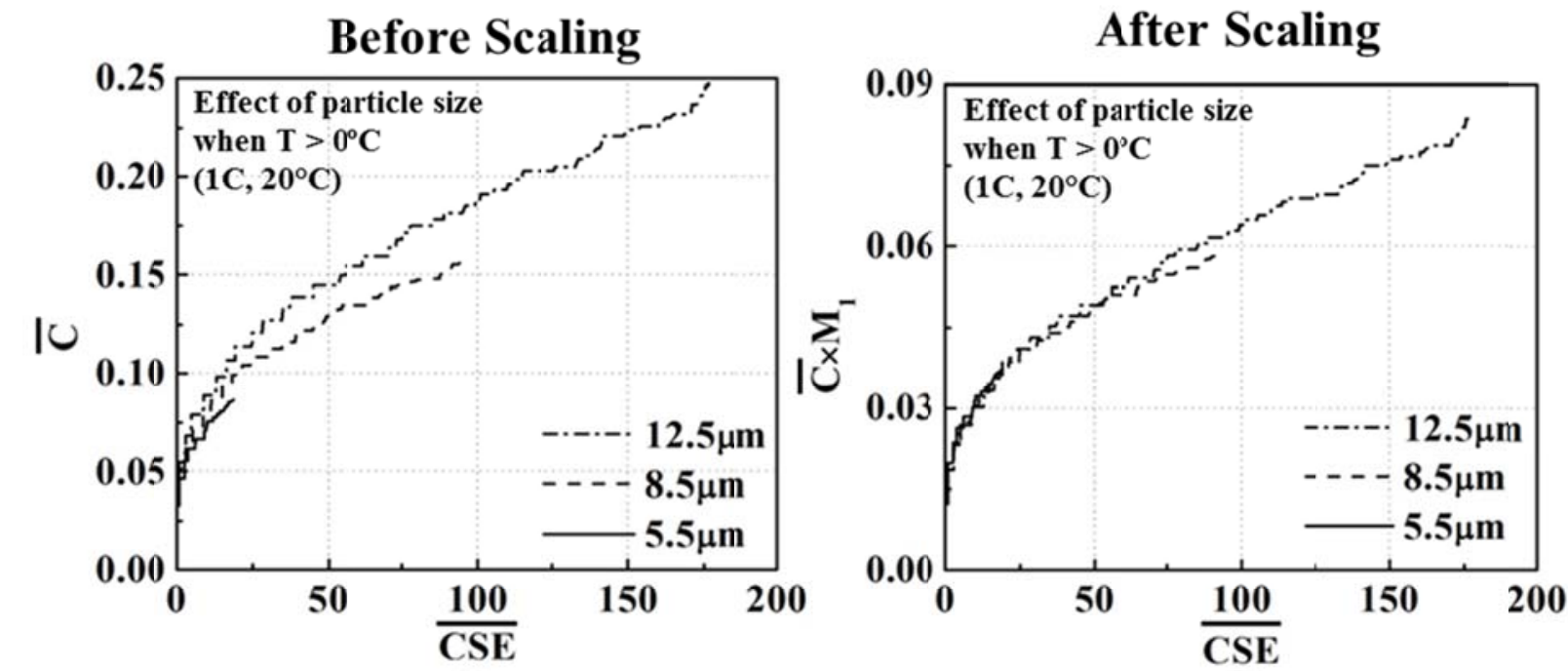

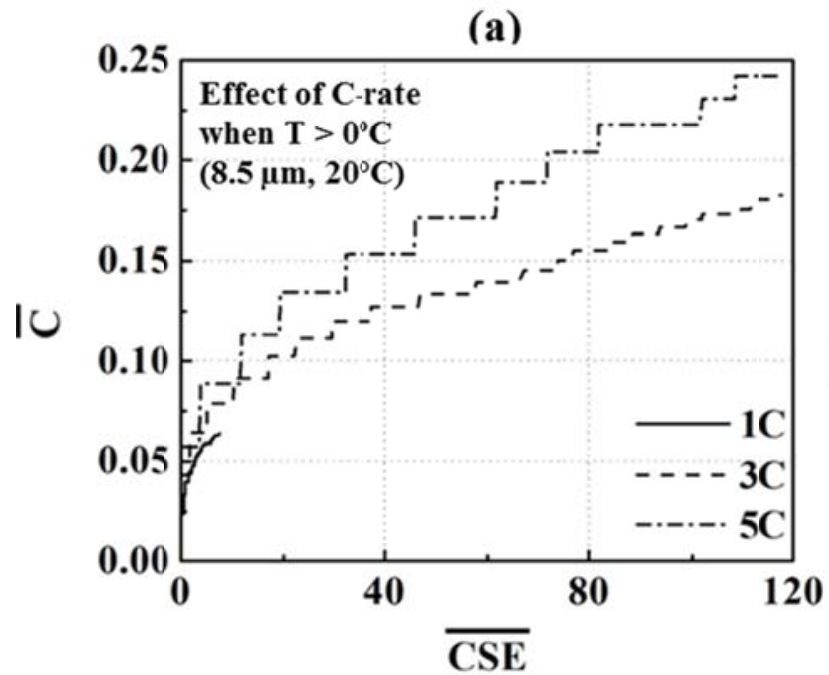

(c)

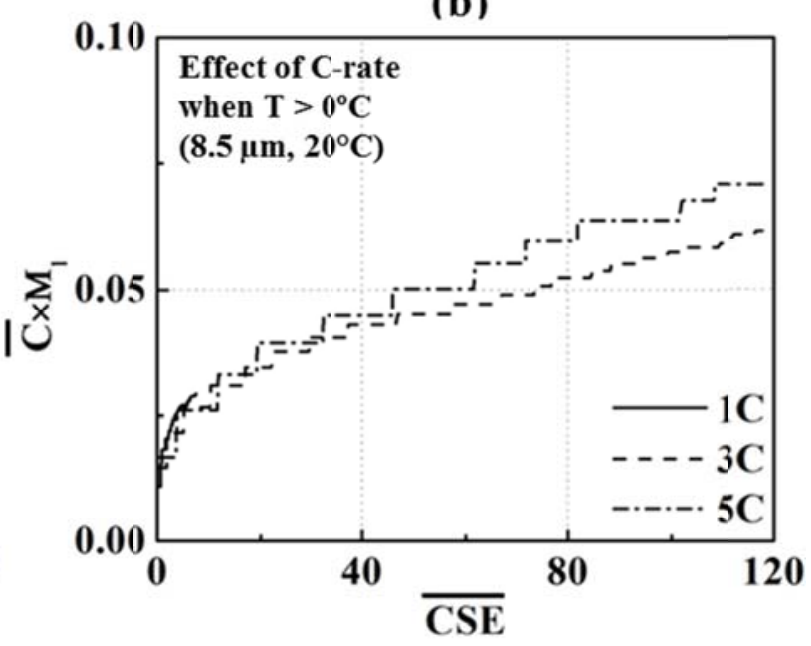

(d)

Figure 2. Relation between cumulative strain energy and concentration gradient for (a) different particle sizes at $1 \mathrm{C}$-rate and $20^{\circ} \mathrm{C}$ before multiplying by the scaling factor; (b) different particle sizes at $1 \mathrm{C}$-rate and $20^{\circ} \mathrm{C}$ after multiplying by the scaling factor; (c) different C-rates at $20^{\circ} \mathrm{C}$ before multiplying by the scaling factor with the particle diameter of $8.5 \mu \mathrm{m}$; and (d) different Crates at $20^{\circ} \mathrm{C}$ after multiplying by the scaling factor with the particle diameter of $8.5 \mu \mathrm{m}$. 
Before Scaling

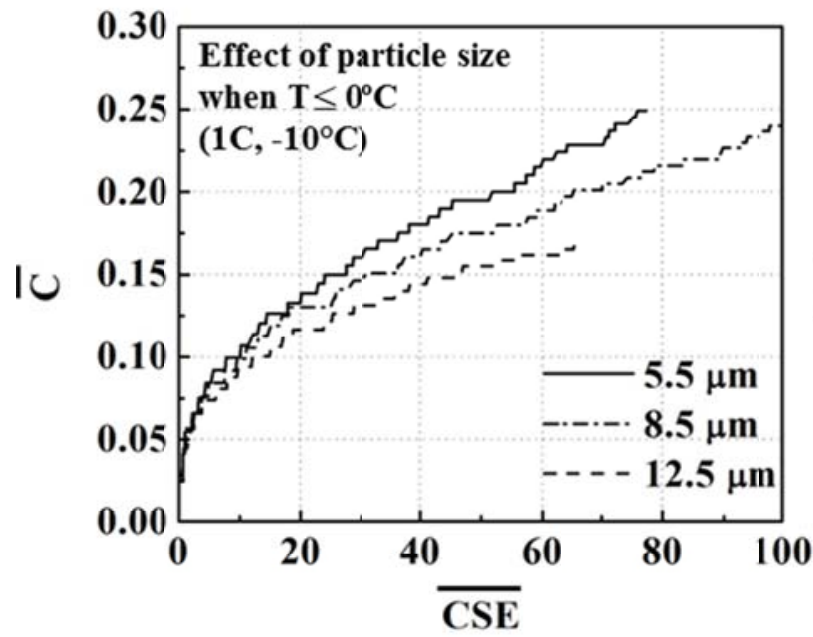

(a)

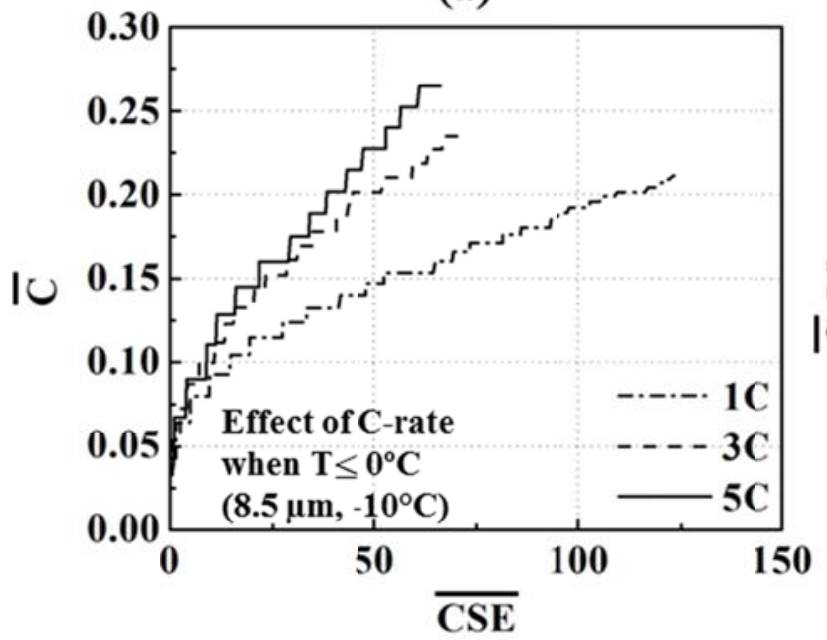

(c)
After Scaling

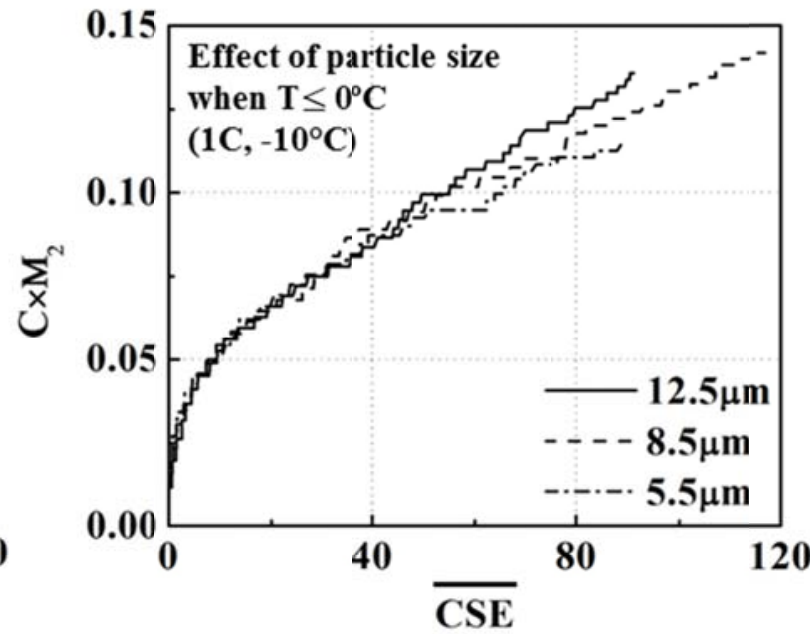

(b)

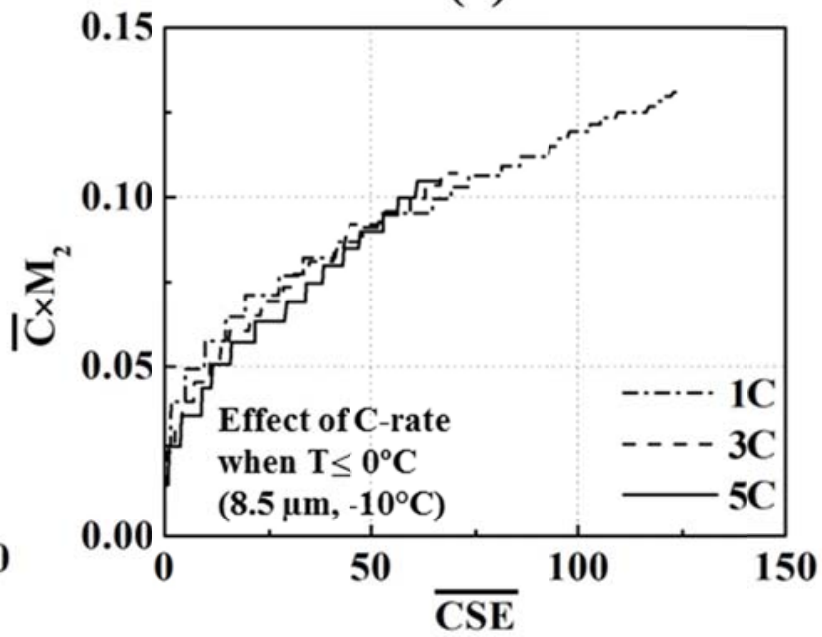

(d)

Figure 3. Relation between cumulative strain energy and concentration gradient for (a) different particle sizes at $1 \mathrm{C}$-rate and $-10^{\circ} \mathrm{C}$ before multiplying by the scaling factor; (b) different particle sizes at $1 \mathrm{C}$-rate and $-10^{\circ} \mathrm{C}$ after multiplying by the scaling factor; (c) different C-rates at $-10^{\circ} \mathrm{C}$ before multiplying by the scaling factor with the particle diameter of $8.5 \mu \mathrm{m}$; and (d) different Crates at $-10^{\circ} \mathrm{C}$ after multiplying by the scaling factor with the particle diameter of $8.5 \mu \mathrm{m}$. 


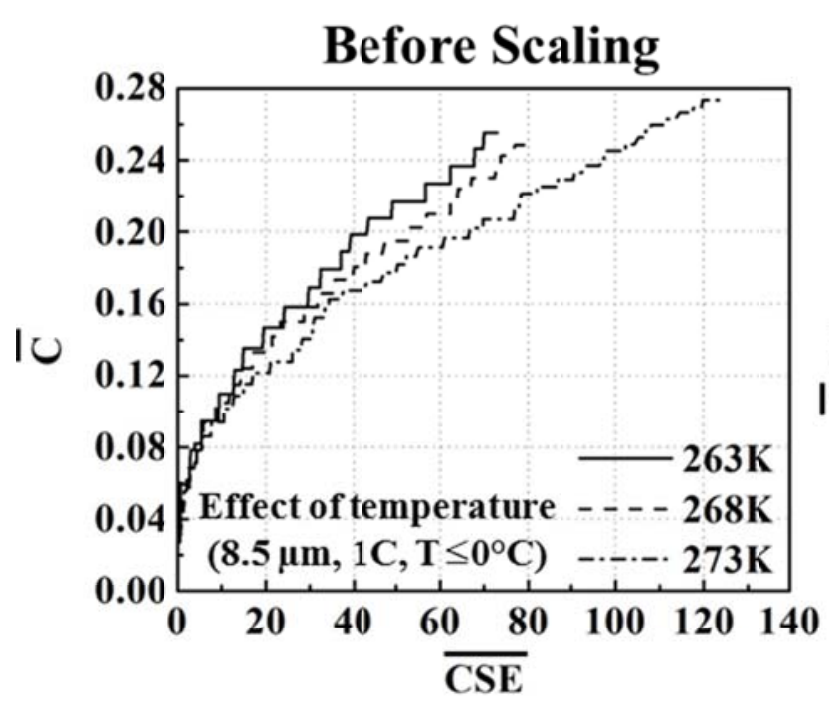

(a)

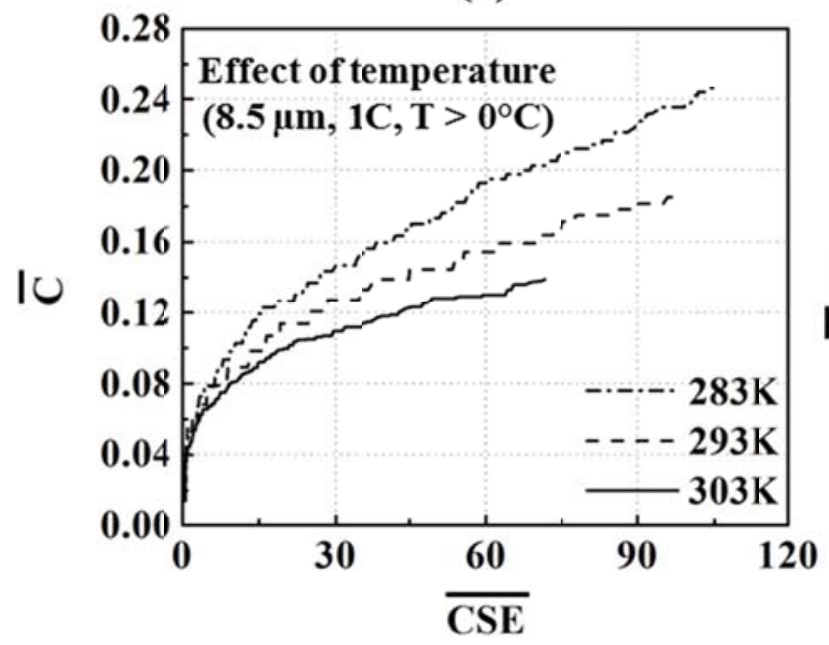

(c)

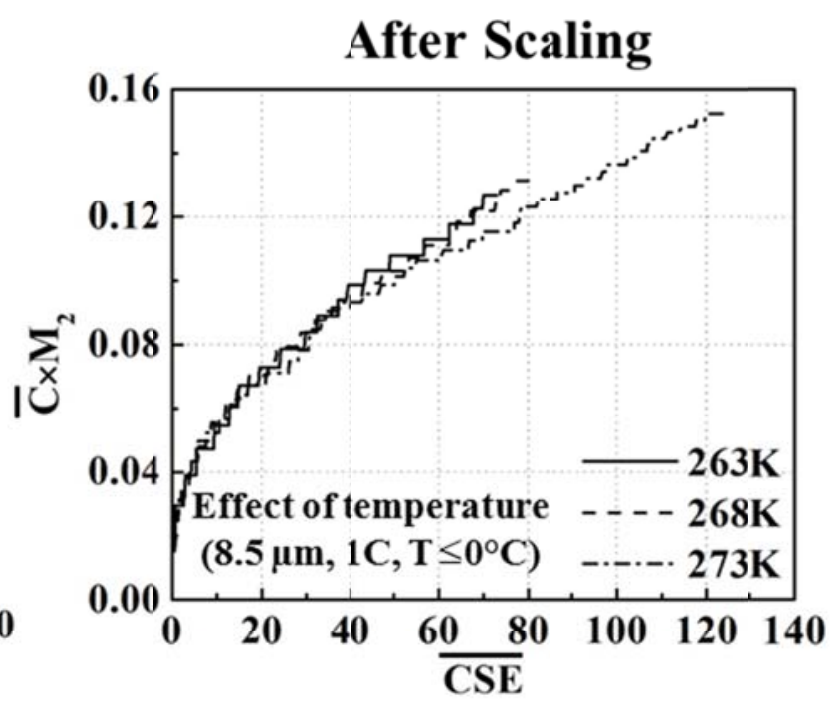

(b)

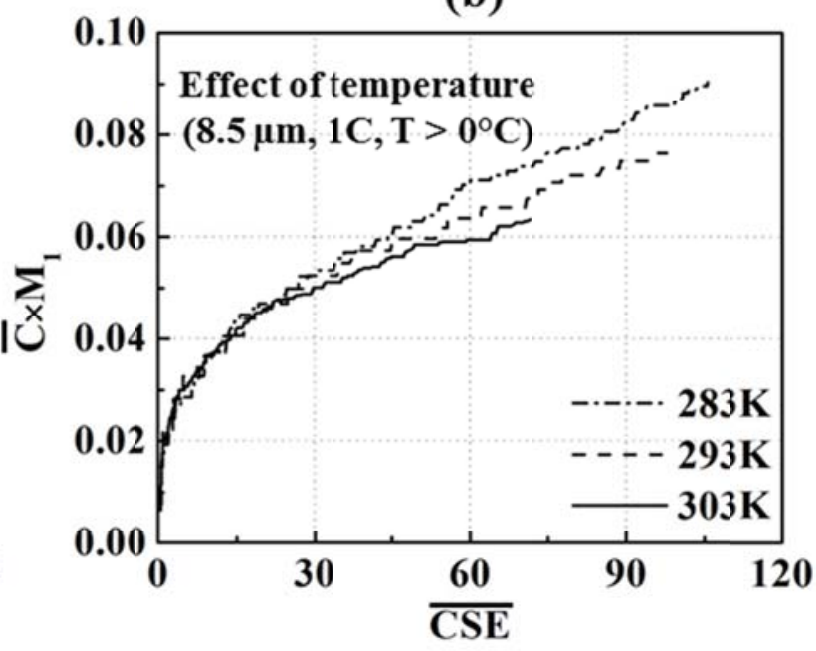

(d)

Figure 4. Relation between cumulative strain energy and concentration gradient for different operation temperatures at $1 \mathrm{C}$-rate with the particle diameter of $8.5 \mu \mathrm{m}$. (a) $\mathrm{T} \leq 0^{\circ} \mathrm{C}$, before multiplying by the scaling factor. (b) $\mathrm{T} \leq 0^{\circ} \mathrm{C}$, after multiplying by the scaling factor. (c) $\mathrm{T}>$ $0^{\circ} \mathrm{C}$, before multiplying by the scaling factor. (d) $\mathrm{T}>0^{\circ} \mathrm{C}$, after multiplying by the scaling factor. 


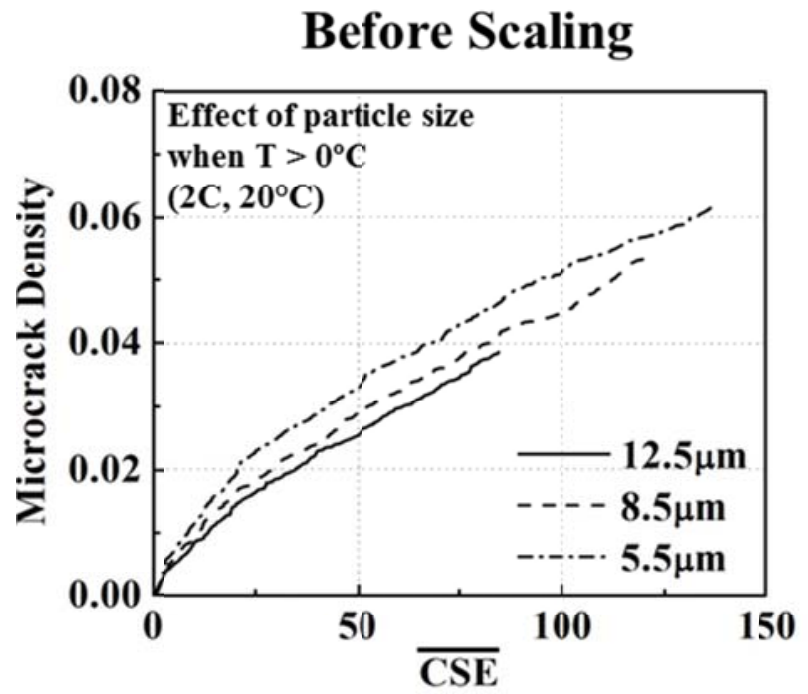

(a)

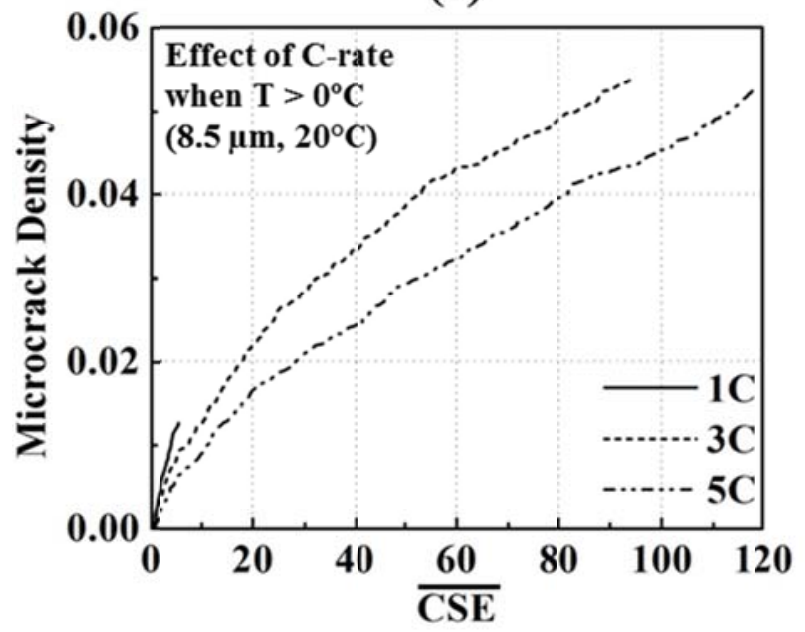

(c)

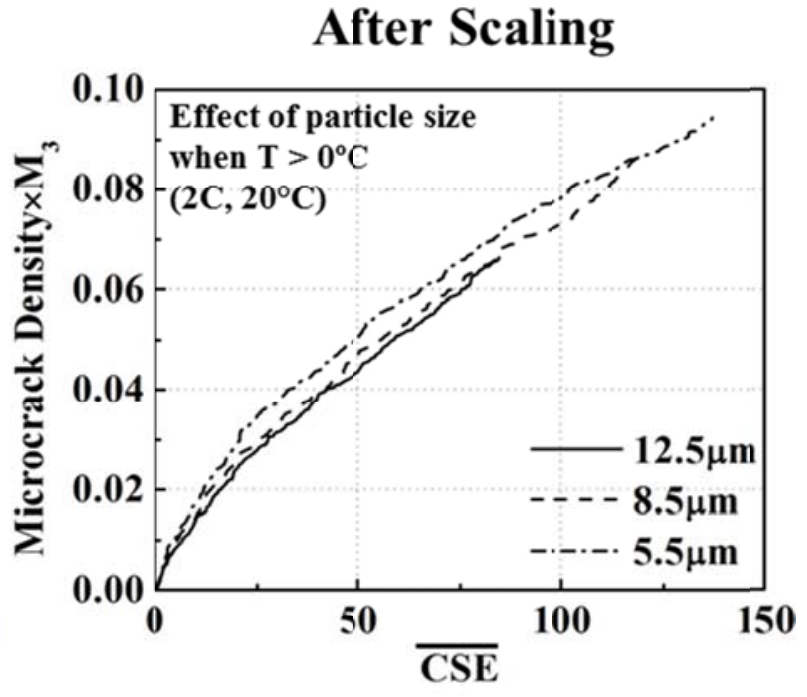

(b)

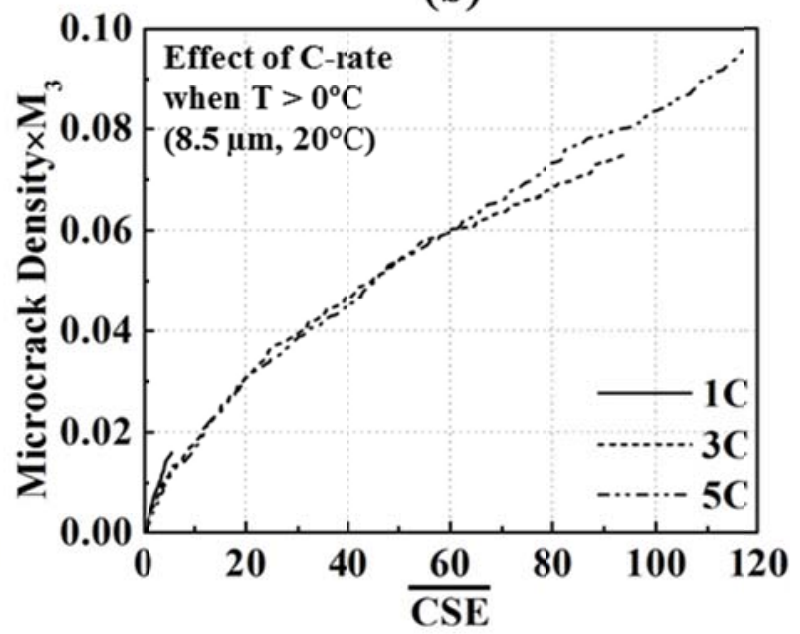

(d)

Figure 5. Relation between cumulative strain energy and microcrack density for (a) different particle sizes at $2 \mathrm{C}$-rate and $20^{\circ} \mathrm{C}$ before multiplying by the scaling factor; (b) different particle sizes at $2 \mathrm{C}$-rate and $20^{\circ} \mathrm{C}$ after multiplying by the scaling factor; (c) different C-rates at $20^{\circ} \mathrm{C}$ before multiplying by the scaling factor with the particle diameter of $8.5 \mu \mathrm{m}$; and (d) different Crates at $20^{\circ} \mathrm{C}$ after multiplying by the scaling factor with the particle diameter of $8.5 \mu \mathrm{m}$. 
Before Scaling

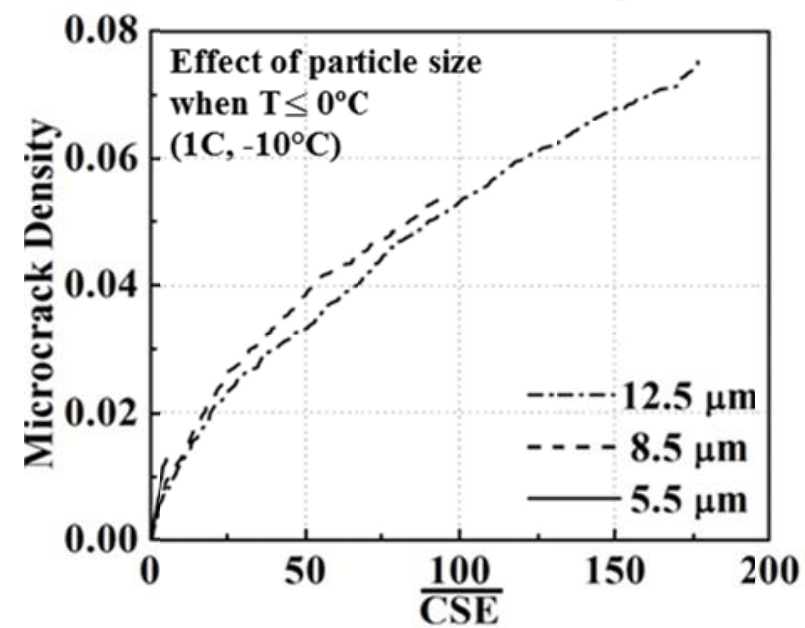

(a)

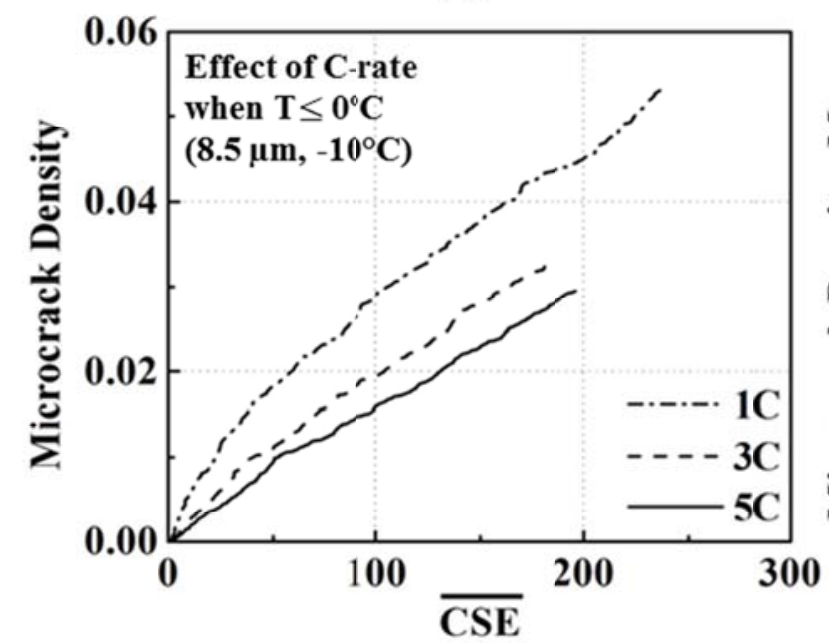

(c)

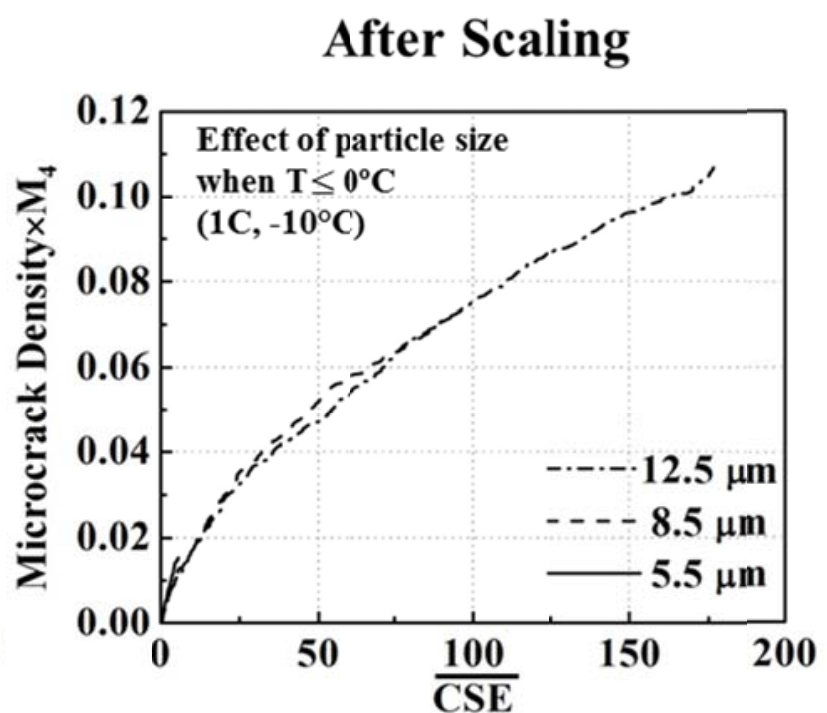

(b)

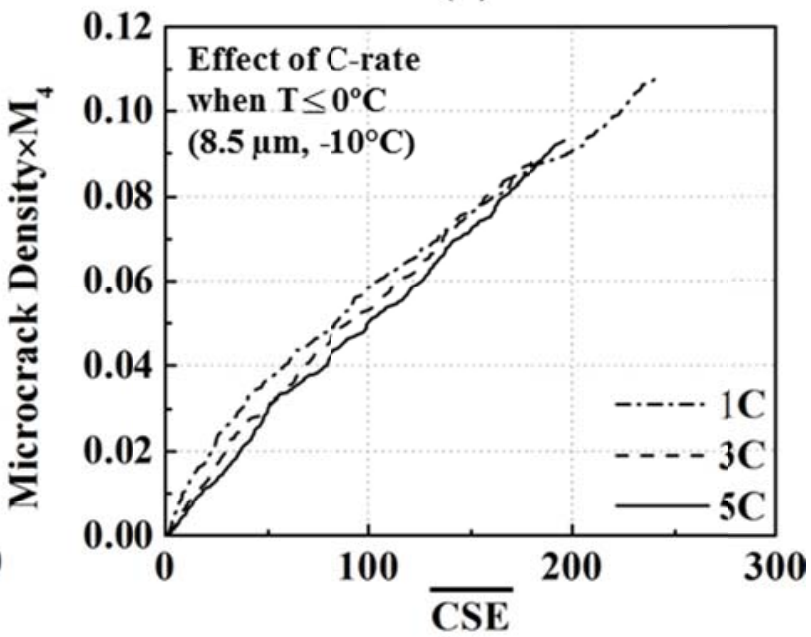

(d)

Figure 6. Relation between cumulative strain energy and microcrack density for (a) different particle sizes at $1 \mathrm{C}$-rate and $-10^{\circ} \mathrm{C}$ before multiplying by the scaling factor; (b) different particle sizes at $1 \mathrm{C}$-rate and $-10^{\circ} \mathrm{C}$ after multiplying by the scaling factor; (c) different C-rates at $-10^{\circ} \mathrm{C}$ before multiplying by the scaling factor with the particle diameter of $8.5 \mu \mathrm{m}$; and (d) different Crates at $-10^{\circ} \mathrm{C}$ after multiplying by the scaling factor with the particle diameter of $8.5 \mu \mathrm{m}$. 


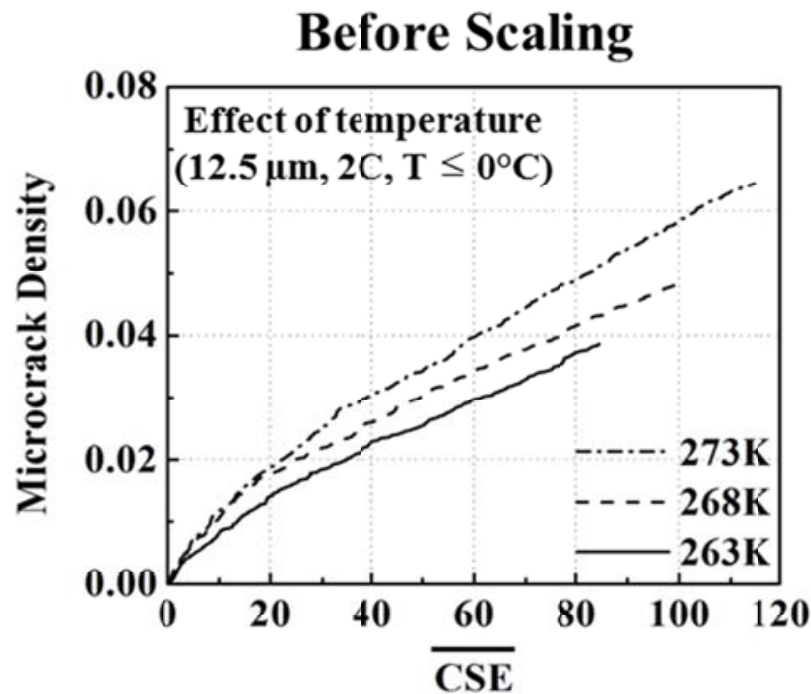

(a)

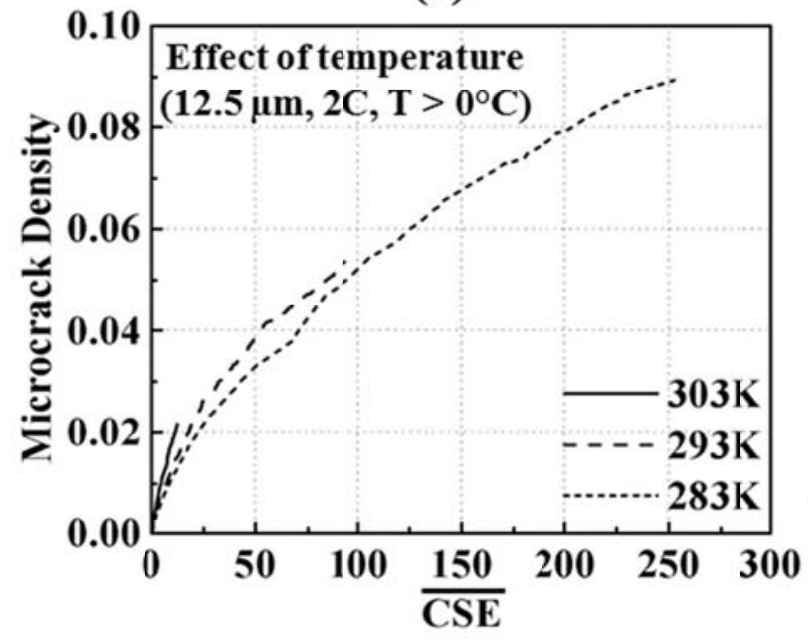

(c)

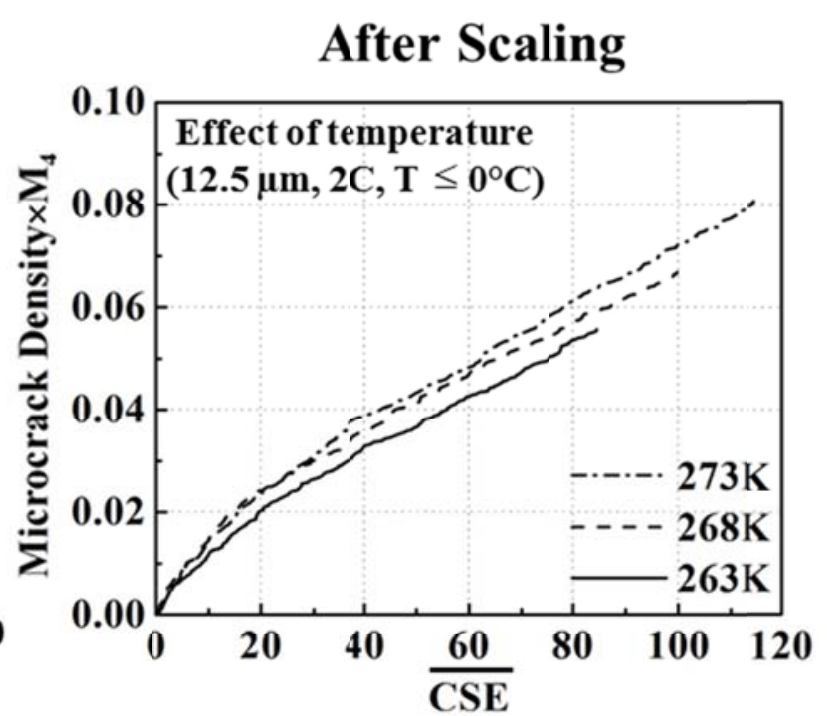

(b)

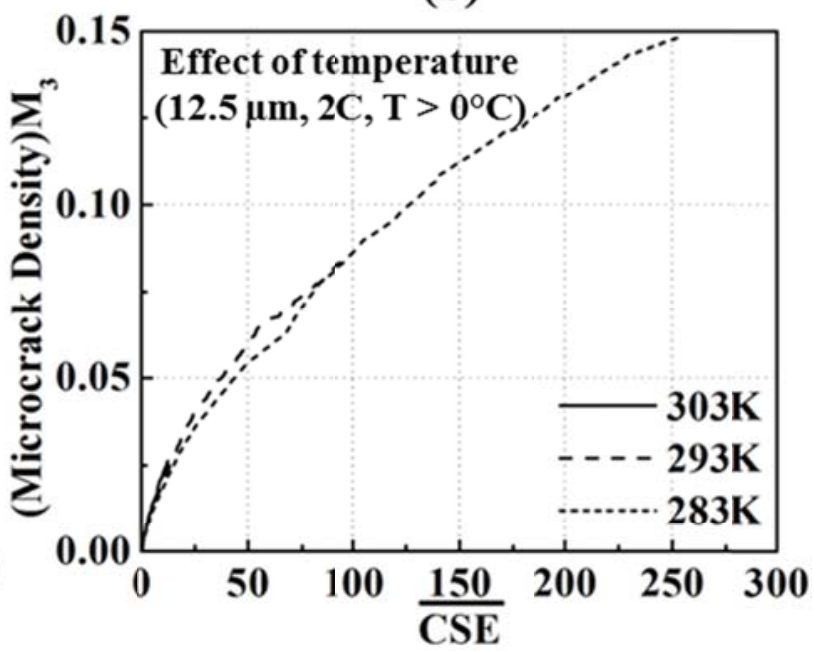

(d)

Figure 7. Relation between cumulative strain energy and concentration gradient for different operation temperatures at $2 \mathrm{C}$-rate with the particle diameter of $12.5 \mu \mathrm{m}$. (a) $\mathrm{T} \leq 0^{\circ} \mathrm{C}$, before multiplying by the scaling factor. (b) $\mathrm{T} \leq 0^{\circ} \mathrm{C}$, after multiplying by the scaling factor. (c) $\mathrm{T}>$ $0^{\circ} \mathrm{C}$, before multiplying by the scaling factor. (d) $\mathrm{T}>0^{\circ} \mathrm{C}$, after multiplying by the scaling factor. 


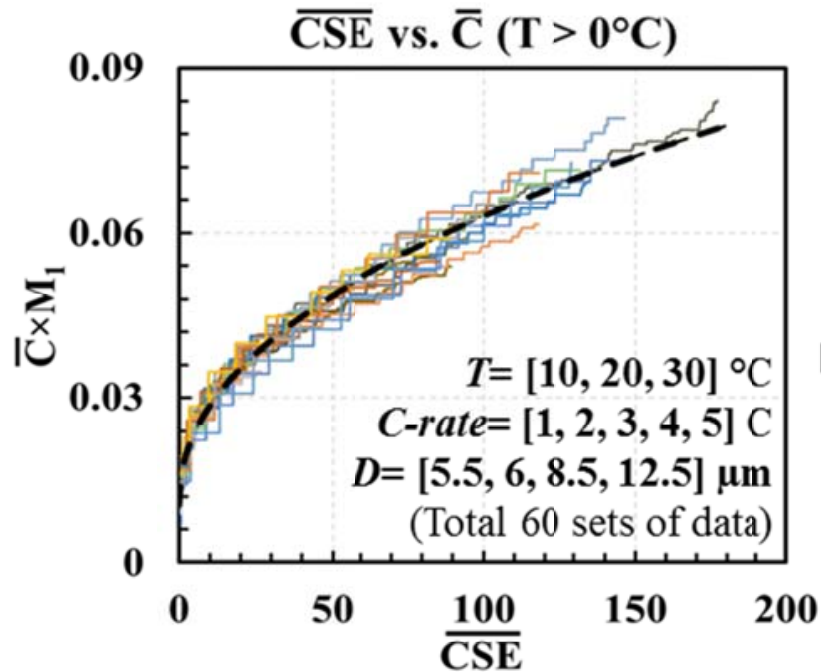

(a)

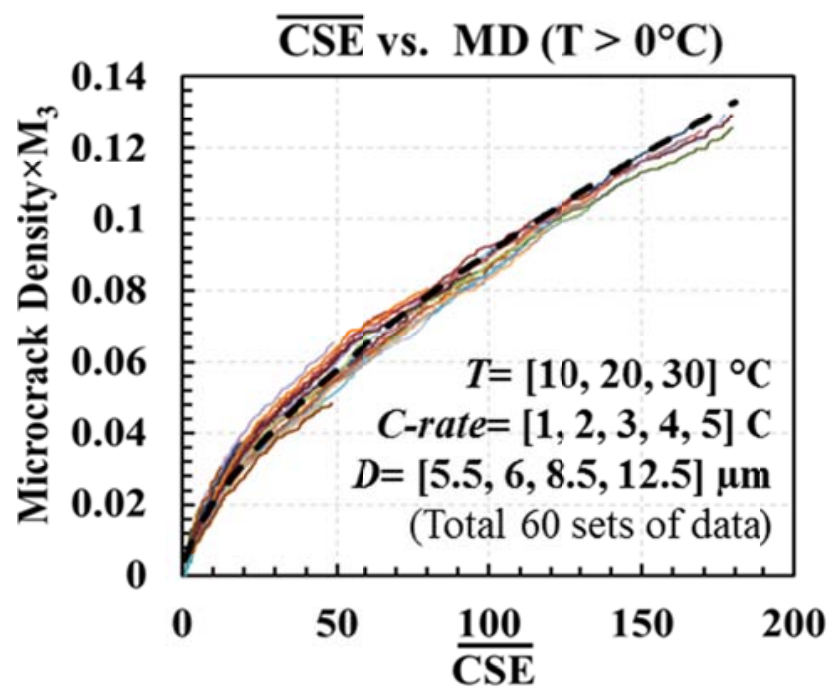

(c)

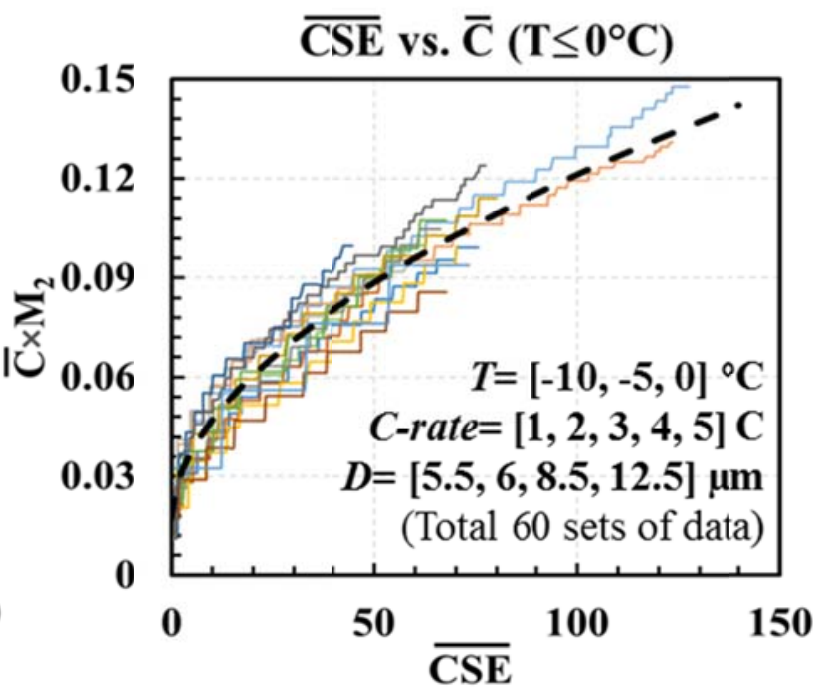

(b)

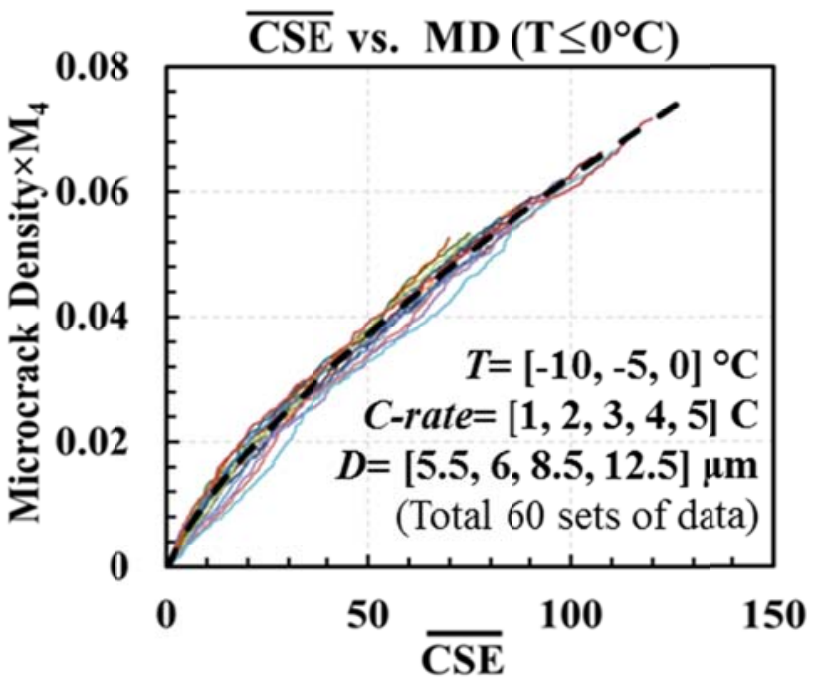

(d)

Figure 8. The fitting result for (a) relation between cumulative strain energy and concentration gradient when $\mathrm{T}>0^{\circ} \mathrm{C}$; (b) relation between cumulative strain energy and concentration gradient when $\mathrm{T} \leq 0^{\circ} \mathrm{C}$; (c) relation between cumulative strain energy and microcrack density when $\mathrm{T}>$ $0^{\circ} \mathrm{C}$; and (d) relation between cumulative strain energy and microcrack density when $\mathrm{T} \leq 0^{\circ} \mathrm{C}$. The dashed line shows the fitting result. 


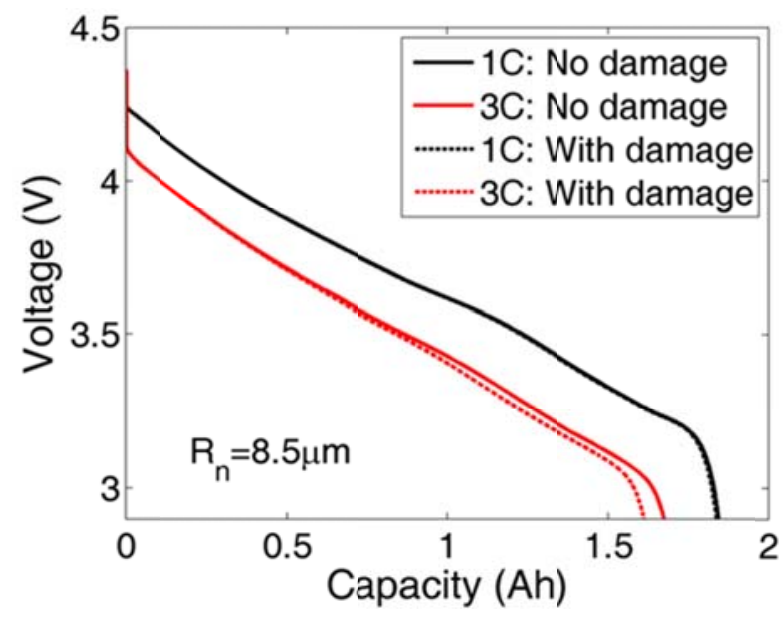

(a)

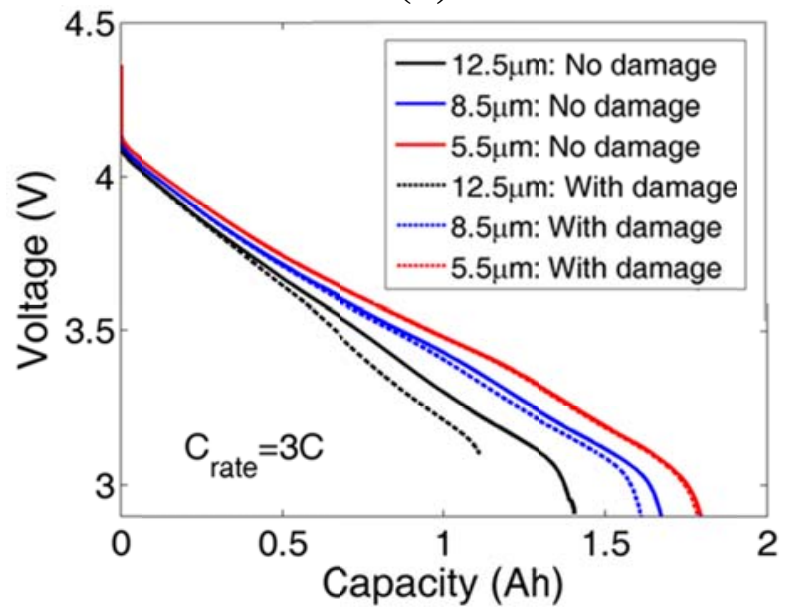

(c)

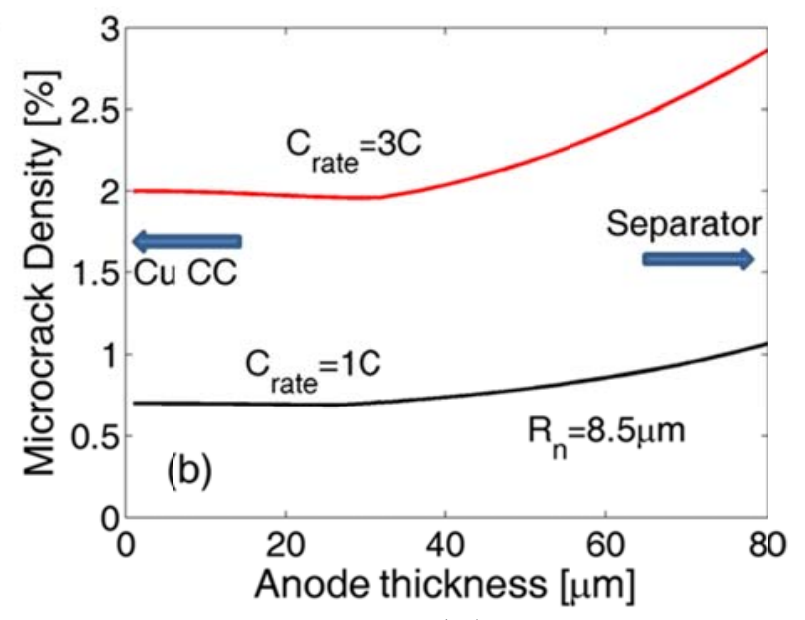

(b)

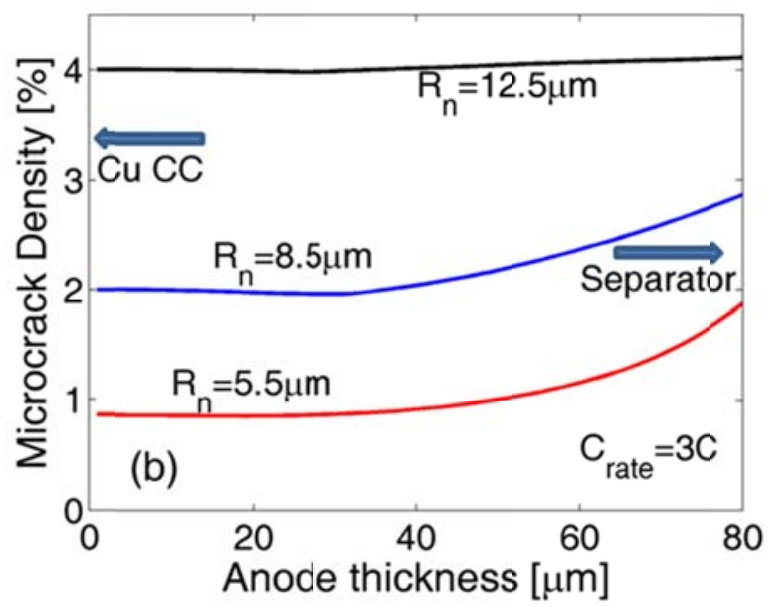

(d)

Figure 9. (a) Performance at different C-rates with and without taking damage evolution into account. Capacity fade is due to mechanical degradation is more prominent at higher C-rates. (b) Distribution of microcrack density at the end of the discharge process of different C-rates. Higher mechanical degradation is observed at larger C-rates. (c) Performance curves for three different particle sizes with and without taking the effect of microcrack evolution into account. Enhanced capacity fade is observed in large sized particles. (d) Evolution of mechanical degradation is more for large sized particles. For small particles $(5.5 \mu \mathrm{m}$ and $8.5 \mu \mathrm{m})$, larger microcrack density evolves near the separator. However, large sized particles experience almost uniform mechanical degradation throughout the anode thickness. 


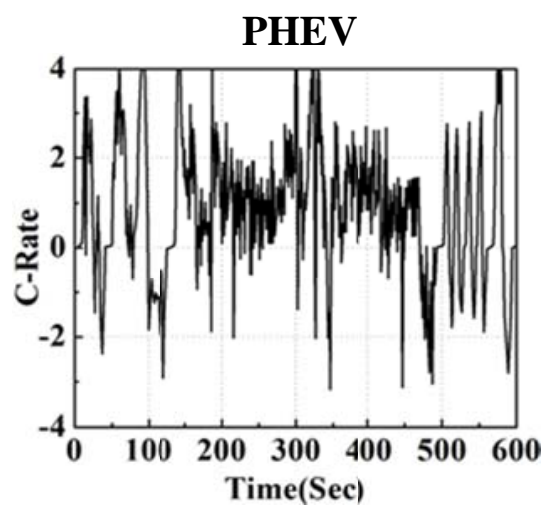

(a)

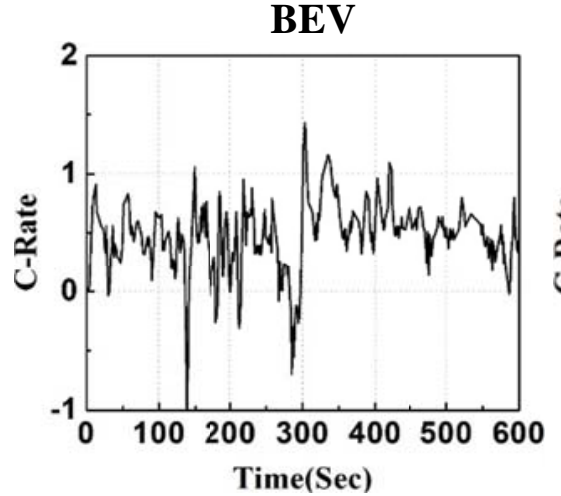

(b)

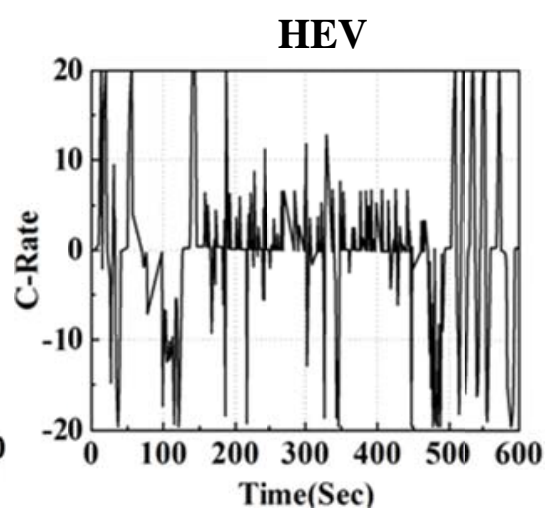

(c)

Figure 10. (a) Variation of C-rate over time for the PHEV drive cycle. (b) Variation of C-rate over time for the BEV drive cycle. (c) Variation of C-rate over time for the HEV drive cycle. 

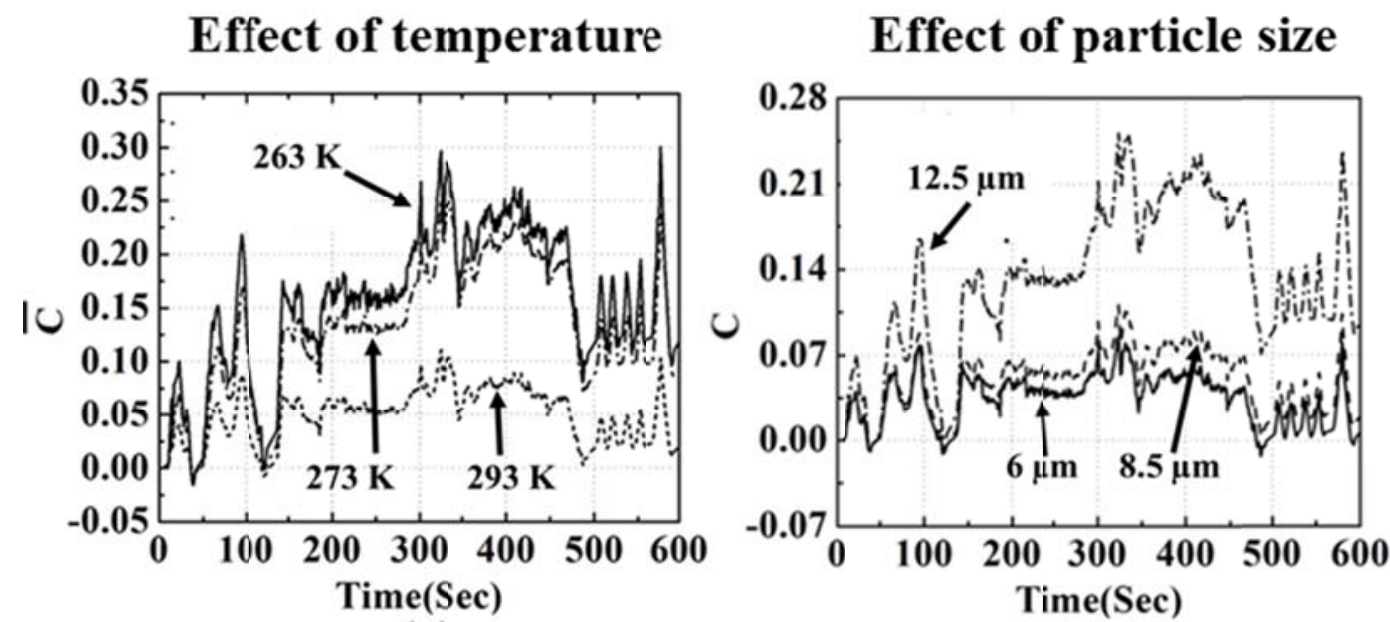

(a)

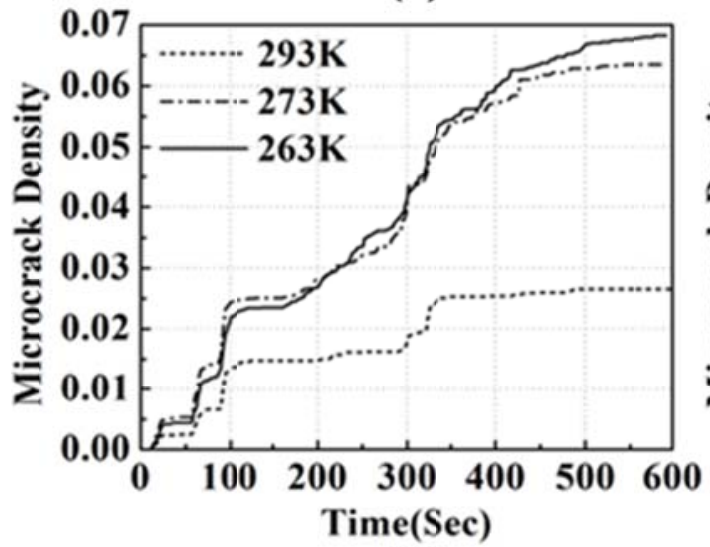

(b)

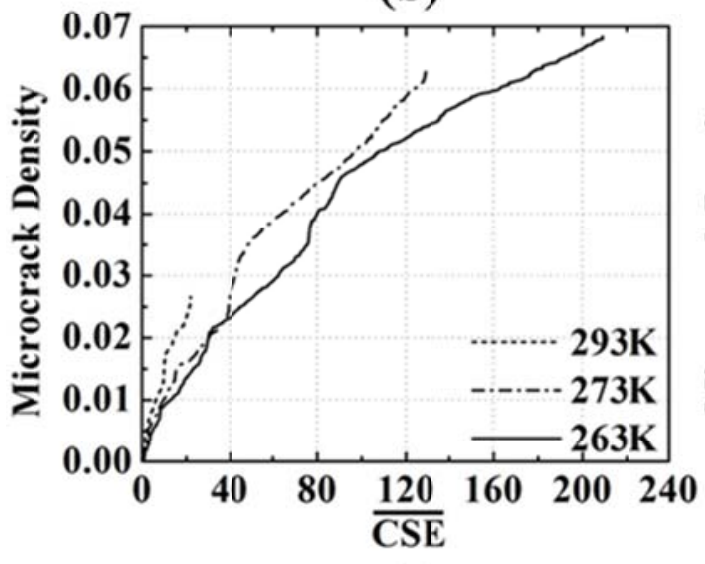

(c)

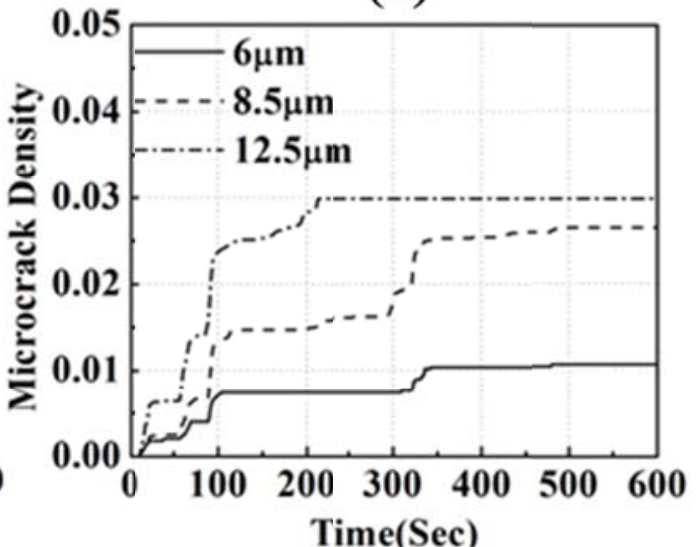

(e)

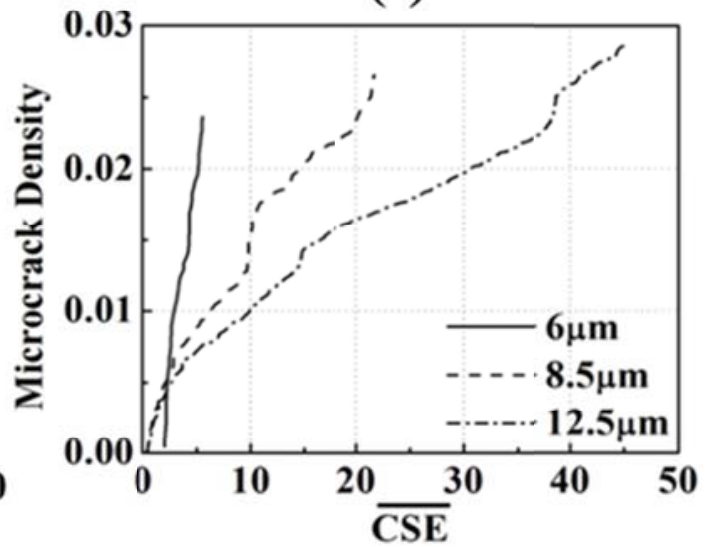

(f)

Figure 11. $(\mathrm{a}-\mathrm{c})$ The influence of temperature on the PHEV drive cycle with particle diameter of $8.5 \mu \mathrm{m}$. (a) The time evolution of the concentration gradient. (b) The time evolution of microcrack density. (c) The relation between microcrack density and CSE. (d-f) The influence of particle size on the PHEV drive cycle at $T=20^{\circ} \mathrm{C}$. (d) The time evolution of the concentration gradient. (e) The time evolution of microcrack density. (f) The relation between microcrack density and CSE. 

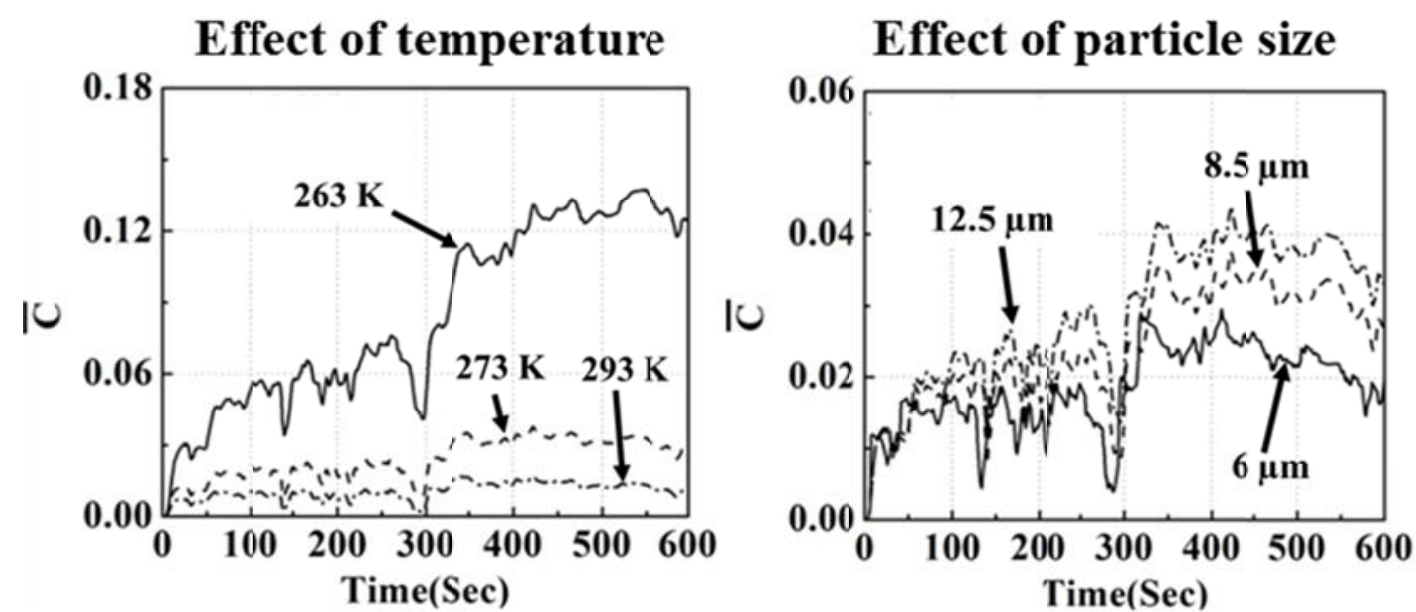

(a)

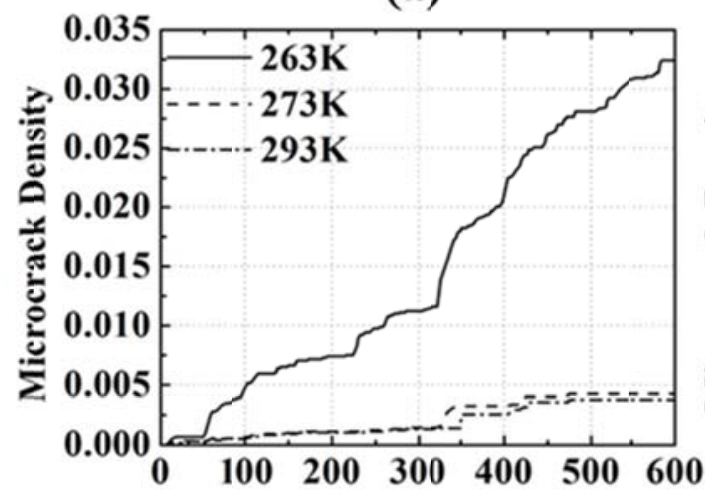

Time(Sec)

(b)

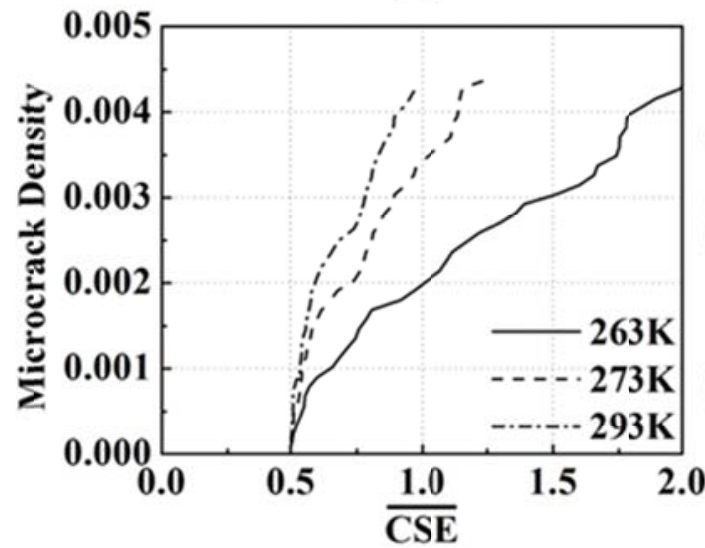

(c)

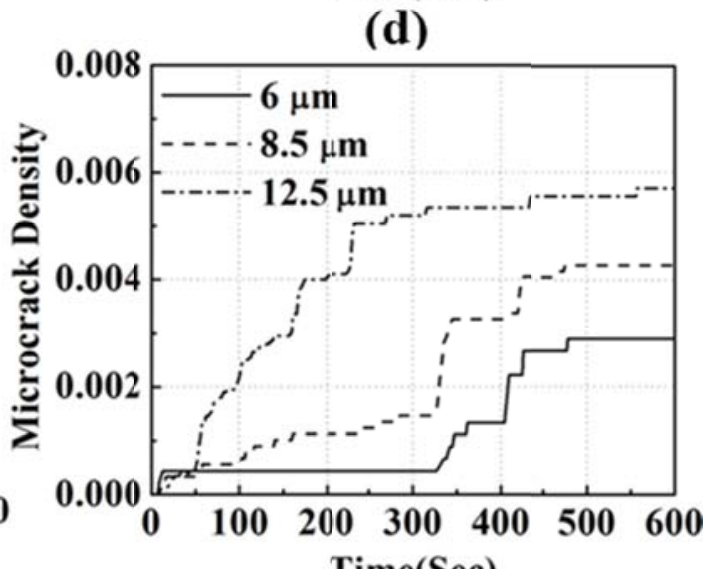

Time(Sec)

(e)

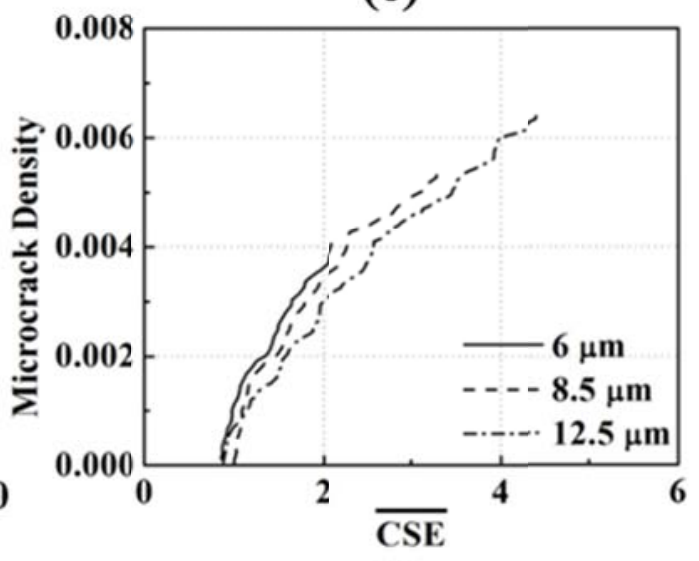

(f)

Figure 12. $(\mathrm{a}-\mathrm{c})$ The influence of temperature on the BEV drive cycle with particle diameter of $8.5 \mu \mathrm{m}$. (a) The time evolution of the concentration gradient. (b) The time evolution of microcrack density. (c) The relation between microcrack density and CSE. (d-f) The influence of particle size on the BEV drive cycle at $T=20^{\circ} \mathrm{C}$. (d) The time evolution of the concentration gradient. (e) The time evolution of microcrack density. (f) The relation between microcrack density and CSE. 

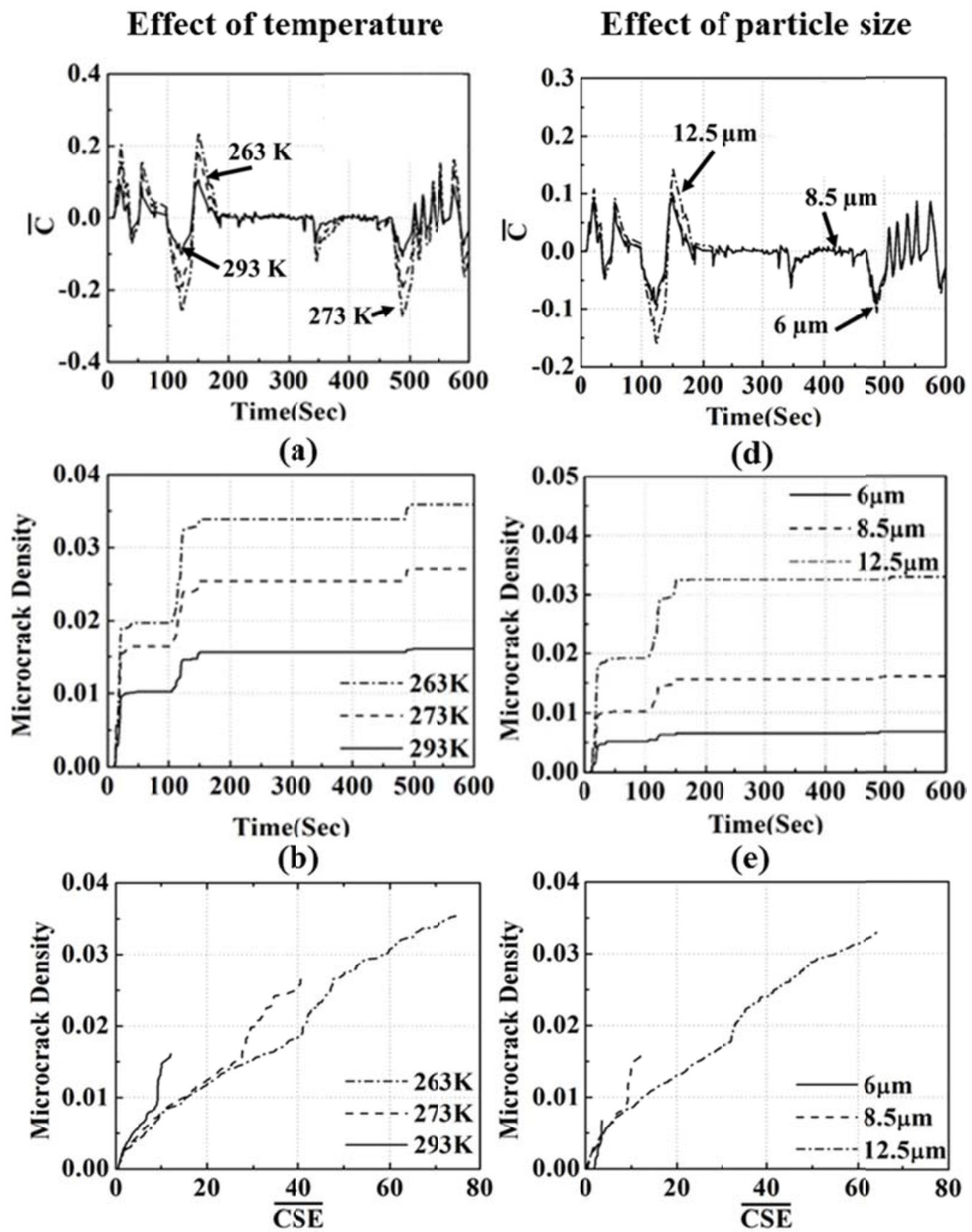

(c)

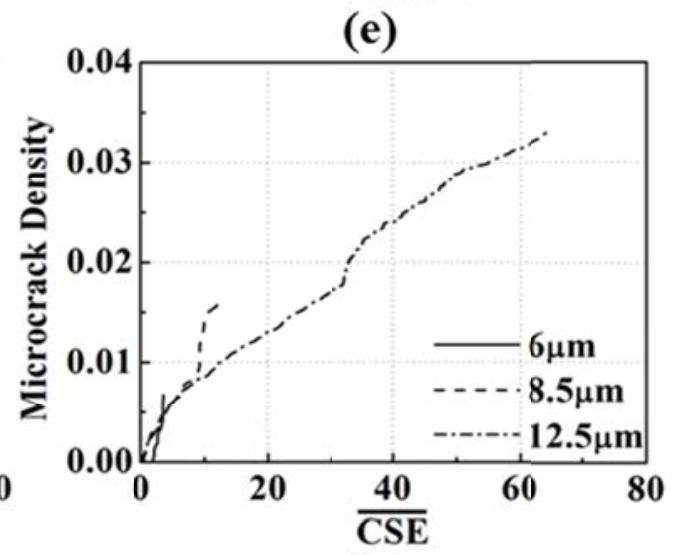

(f)

Figure 13. $(a-c)$ The influence of temperature on the HEV drive cycle with particle diameter of $8.5 \mu \mathrm{m}$. (a) The time evolution of the concentration gradient. (b) The time evolution of microcrack density. (c) The relation between microcrack density and CSE. (d-f) The influence of particle size on the HEV drive cycle at $T=20^{\circ} \mathrm{C}$. (d) The time evolution of the concentration gradient. (The results of particle size $6 \mu \mathrm{m}$ and $8.5 \mu \mathrm{m}$ almost overlap). (e) The time evolution of microcrack density. (f) The relation between microcrack density and CSE. 


\section{PHEV}

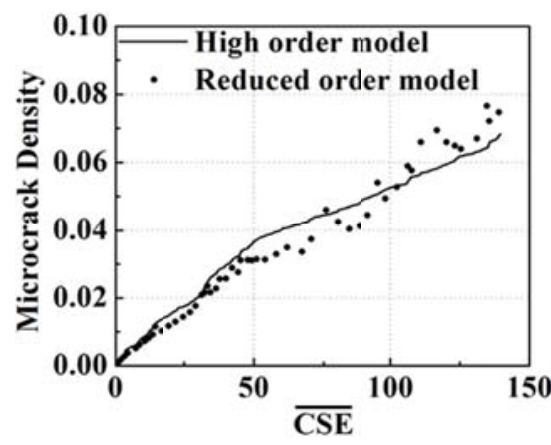

(a)

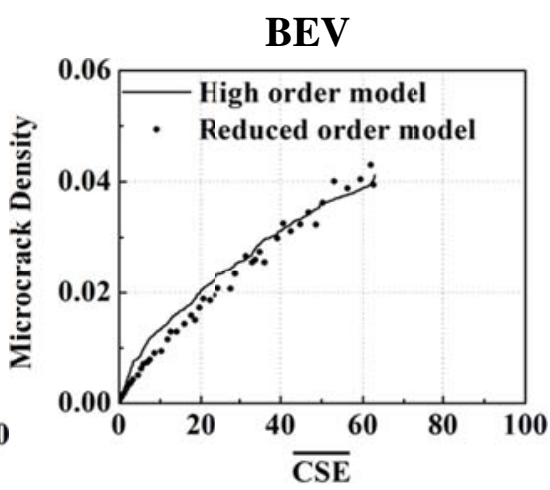

(b)

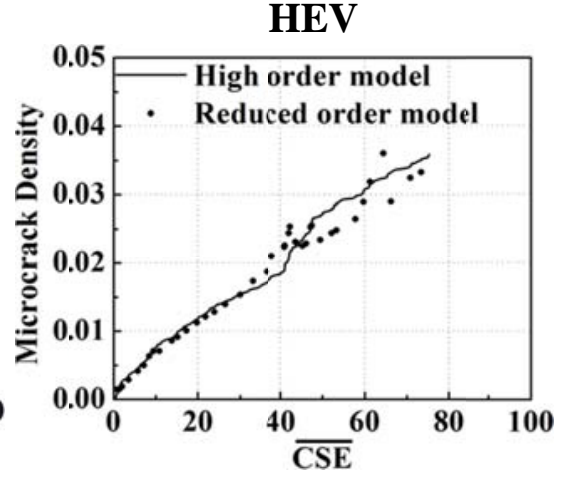

(c)

Figure 14. Comparison of the relation between microcrack density and CSE between high order diffusion induced damage model and reduced order relation in Eq. (13). (a) PHEV drive cycle with $8.5 \mu \mathrm{m}$ particle under $-10^{\circ} \mathrm{C}$. (b) $\mathrm{BEV}$ drive cycle with $8.5 \mu \mathrm{m}$ particle under $-10^{\circ} \mathrm{C}$. (c) $\mathrm{HEV}$ drive cycle with $8.5 \mu \mathrm{m}$ particle under $-10^{\circ} \mathrm{C}$. 